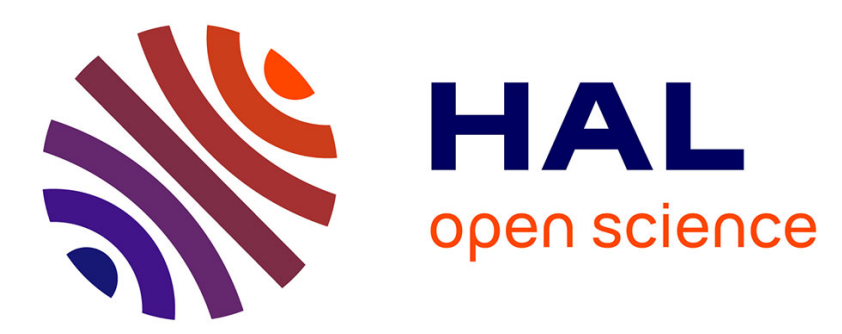

\title{
On the stability analysis of boundary conditions for the wave equation by energy methods. Part I: the homogeneous case
}

\author{
T. Ha-Duong, Patrick Joly
}

\section{- To cite this version:}

T. Ha-Duong, Patrick Joly. On the stability analysis of boundary conditions for the wave equation by energy methods. Part I : the homogeneous case. RR-1306, INRIA. 1990. inria-00075253

\section{HAL Id: inria-00075253 \\ https://hal.inria.fr/inria-00075253}

Submitted on 24 May 2006

HAL is a multi-disciplinary open access archive for the deposit and dissemination of scientific research documents, whether they are published or not. The documents may come from teaching and research institutions in France or abroad, or from public or private research centers.
L'archive ouverte pluridisciplinaire HAL, est destinée au dépôt et à la diffusion de documents scientifiques de niveau recherche, publiés ou non, émanant des établissements d'enseignement et de recherche français ou étrangers, des laboratoires publics ou privés. 


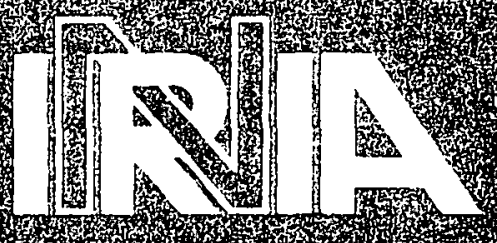

\section{Rapports de Recherche}

464.

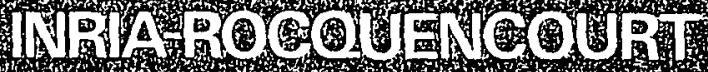

\%

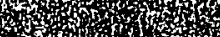

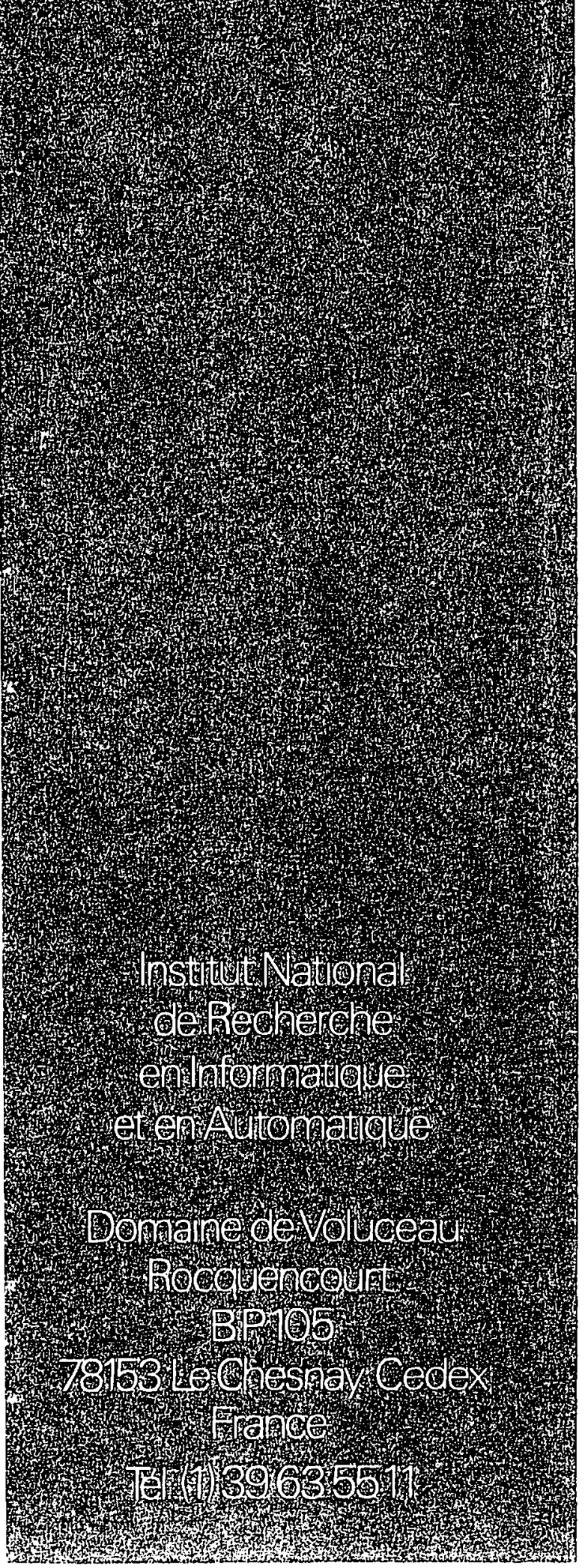


On the stability analysis of boundary conditions

for the wave equation by energy methods

Part I : The homogeneous case

T. HA-DUONG ${ }^{1}$ and P. JOL $Y^{2}$

1 Ecole Polytechnique

C.M.A.P.

91128 Palaiseau

2 INRIA

Domaine de Voluceau

78153 Le Chesnay Cedex 


\section{On the stability analysis of boundary conditions \\ for the wave equation by energy methods \\ Part I : The homogeneous case}

Tuong HA-DUONG 1 and Patrick JOLY ${ }^{2}$

Astract:

We reconsider the stability theory of boundary conditions for the wave equation from the point of view of energy techniques. We study here, for the case of the homogeneous half-space, a large class of boundary conditions including the so called absorbing conditions. We show that the results of strong stability in the sense of Kreiss, studied from the point of view of the modal analysis by Trefethen Halpern, always correspond to the decay in tme of a particular energy. This result leads to the obtention of new estimates for the solution of the associated mixed problem.

Sur l'analyse de stabilité de conditions aux limites pour l'équation des ondes par la méthode énergétique.

Partie I: le cas homogène

Résumé:

Nous réexaminons la théorie de la stabilité des conditions aux limites pour l'équation des ondes du point de vue des techniques énergétiques. Nous étudions ici, dans le cas du demi espace homogène, une large classe de conditions aux limites incluant les conditions dites absoriantes. Nous montrons que les résultats de stabilité forte au sens de Kreiss, érudiés du point de vie de l'analyse modale par Trefethen-Halpern, correspondent toujours à la décroissance d'une certaine énergie. Ce résultat mène à l'obrention de nouvelles estimations sur la solution du problème mixie associé.

Mots clé : Equation des ondes - Conditions aux limites - Problèmes hyperboliques mixtes Théorie de la stabilité - Estrmations d'énergie.

Kev words: Wave equation - Boundary conditions - Mixed hyperbolic problems - Stability theory - Energy estimates.

1. CMAP, Ecole Polytechnique, 91128 Paiaiseau Cedex;

2. INRIA, Domaine de Voluceau, 78153 Le Chesnay Cedex. 


\section{INTRODUCTION AND PRESENTATION OF THE PAPER}

The theory of the stability of initial boundary value problems for hyperbolic systems, has known an important development at the end of the 70's with the major work of Kreiss [K]. A very interesting review paper of this theory has been recently given by Higdon in [Hi].

It appears that the relative complexity of this theory comes from the difficulty of a good definition for the stability or well-posedness of these problems and from the technical character of the proofs. In fact one can roughly distinguish two kinds of stability definitions, namely :

- the weak well-posedness (or weak stability)

- the strong well-posedness (or strong stability).

The weak well-posedness corresponds to the classical well-posedness in the sense of Hadamard, means that there is existence and uniqueness of the solution and that one can estimate some norm (let us say of Sobolev type) of the solution by some norm of the data which implies, since the equations are linear, that the application data $\rightarrow$ solution is continuous for appropriate topologies. In its definition of the strong stability, Kreiss prescribes a priori the norms for which he wants to obtain some estimates, which is of course a stronger result.

For instance if one considers the Cauchy problem whose initial conditions are the only data, one requires that the $\mathrm{L}^{2}$ norm (in space and time, including the trace on the boundary) of the solution must be estimated (modulo a constant which may depend on the interval of time $[0, T]$ one considers) by the $\mathrm{L}^{2}$ norm of the initial data : there is no loss of derivatives. On the other hand, the problem is weakly well-posed as soon as one can control the same $\mathrm{L}^{2}$ norm of the solution by some higher Sobolev norm of the solution. We shall give a simple concrete illustration of the distinction between these two notions in this article (see section 2.2).

In the case of a half-space of $\mathbb{R}^{d}$, the two types of stability are studied for first order strictly hyperbolic systems with constant coefficients via the so-called modal analysis which means that one uses the Laplace-transform in time and a Fourier-transform with respect to the variables tangent to the boundary of the half-space. This study leads to the concepts of generalized eigenvalues and characteristic equation which are extensively discussed in [Hi] with a geometrical interpretation, using characteristic manifolds and group velocities. We shall not enter the details here. Let us simply mention that the characteristic equation takes the form :

$$
F(s, \theta)=0
$$


where $s \in \mathbb{C}$ is the unknown and $\theta$ belongs to the unit sphere of $\mathbb{R}^{\mathrm{d}-1}$. Once the characteristic equation (1.1), which is a polynomial equation with respect to $s$, has been determined, the difficulty is reduced in the location of the solutions of this equation in the complex plane. The case of weakly well-posed problems corresponds to the result of Hersch [He] while the case of strongly well-posed problems corresponds to the result of Kreiss [K]. These results can be summarized as follows :

$$
\left\{\begin{array}{l}
\text { Weak well-posedness } \Leftrightarrow\{\text { solutions of }(1.1)\} \subset\{\mathscr{R} \text { e s } \geq 0\} \\
\text { Strong well-posedness } \Leftrightarrow\{\text { solutions of }(1.1)\} \subset\{\mathscr{R} \text { e s }>0\}
\end{array}\right.
$$

Otherwise, the corresponding problem will be said to be strongly ill-posed. In the last past years, the emphasis has been put on the strong stability notion and the works of Kreiss have been generalized to various situations by different authors (see [Hi], [MO] for instance). One of the reasons is probably the fact that this concept has led to an analogous stability theory for the finite differente approximations to mixed hyperbolic initial boundary value problems (see [GKS], [GT], [Mi], [T] ). The other major interest of the strong well-posedness concept holds in the fact that Kreiss has been able to prove that the stability results could be extended to the case of smooth variable coefficients. Its proof is very complicated and makes use of the theory of symmetrizers and pseudodifferential operators $([\mathrm{K}])$. The precise condition about the variations of the coefficients (which are allowed to vary in space and time) can be stated as follows :

$$
\begin{aligned}
& \text { The coefficients are of class } C^{\infty} \text { with respect to } \\
& \text { space and time variable and are asymptotically } \\
& \text { constant at infinity. }
\end{aligned}
$$

Taking into account the finite velocity propagation of solutions of hyperbolic systems, the constraint that the coefficients are constant at infinity is not really troublesome. The relative weakness of the result lies more in the fact that one cannot say anything in the case where the coefficients are not smooth.

Of course this is due to the technique of proof which uses pseudo-differential operators. It is natural to think to other methods as the energy methods. In most of the systems derived for physical phenomena, one can associate an energy to the solution which can be shown to be conserved in time in the case of the pure initial value problem : these are conservative hyperbolic systems ([F], [W]). The acoustic wave equation, in which we are particularly interested in this paper, belongs to this category of systems. The study of the stability of the wave equation associated to various boundary conditions has a very important practical interest if one thinks for instance to absorbing boundary conditions. In their second paper on the subject, Engquist and Majda [EM] applied the theory of 
Kreiss to show the strong stability of their boundary conditions. More recently, Halpern and Trefethen [TH] considered a completely general class of boundary conditions for the wave equation and, applying Kreiss theory, obtained explicit necessary and sufficient criteria (concerning the coefficients of the differential operator appearing in the boundary condition) for the strong wellposedness of the corresponding initial boundary value problem. We shall state their precise results in the next section. Associated to this work, a question naturally arises : is it possible to obtain the same results by energy estimates?

This is the question we intend to address in this paper. In his review [Hi], Higdon evocated briefly this question and concluded that, if energy techniques fail, this does not mean that the problem is not well-posed but simply that the energy method is not adapted.

As far as we know, the only case where the energy method has been shown to work is the case of the first order absorbing boundary condition.

In the present paper, we are going to show that appropriate energy methods lead to the stability result for a class of boundary conditions which is almost the same as the one considered by Trefethen-Halpern in [TH]. More precisely we shall show, for the model problem of the wave equation in the homogeneous half-space $(\mathrm{N}=2)$, one can show that the strong stability result is associated to the decay in time of some energy associated to the solution. This energy is not necessarily the physical one : by energy, we mean a quadratic form with respect to homogeneous linear differential operators of a given order applied to the solution, which is equal to 0 if and only if the solution $\mathrm{u}$ is identically equal to 0 , provided it vanishes at infinity. More precisely, we shall show that, if one considers the problem:

$$
\begin{cases}\frac{\partial^{2} u}{\partial t^{2}}-\frac{\partial^{2} u}{\partial x^{2}}-\frac{\partial^{2} u}{\partial y^{2}}=0 & x \in \mathbb{R}, y<0, t>0 \\ B_{N} u=0 & x=0, t>0 \\ u(x, y, 0)=u_{d}(x, y) & x=0, y<0 \\ \frac{\partial u}{\partial t}(x, y, 0)=u_{1}(x, y) & \end{cases}
$$

where $B_{N}=B_{N}\left(\frac{\partial}{\partial t}, \frac{\partial}{\partial x}, \frac{\partial}{\partial y}\right)$ is an appropriate homogeneous differential operator of order $N$, the strong well-posedness of (1.2) will correspond to the decay in time of an energy involving $\mathrm{N}^{\text {th }}$ order derivatives of the solution. For instance, the physical energy : 


$$
E(u ; t)=\frac{1}{2} \int_{\Omega}\left|\frac{\partial u}{\partial t}\right|^{2} d x+\frac{1}{2} \int_{\Omega}|\nabla u|^{2} d x
$$

is an energy in our sense for $N=1$. The class of differential operators for which our analysis will be valid is the sub-class of the one considered by Halpern-Trefethen for which direction $\mathrm{x}$ and $-\mathrm{x}$ play the same role, in other words for which $B_{N}=B_{N}\left(\frac{\partial}{\partial t}, \frac{\partial}{\partial y}, \frac{\partial^{2}}{\partial x^{2}}\right)$. For larger dimensional problems, this hypothesis is to be replaced by the invariance of $B_{N}$ with respect to rotations in the tangential hyperplane, thus $B_{N}=B_{N}\left(\frac{\partial}{\partial t}, \frac{\partial}{\partial y}, \Delta_{x}\right)$. We think that this particular class is the most interesting case in view of the practical applications. For such particular conditions, Higdon [Hi2] observed that the stability analysis was essentially reduced to a $1 \mathrm{D}$ analysis. This remark will also be the starting point of our method.

It seems to us that this new approach of the stability theory of boundary conditions associated with the wave equation has the two following advantages :

1) It gives a new enlightening to the Kreiss theory applied to the wave equation. Moreover it leads to results which are stronger than those simply deduced from the direct application of Kreiss theorem in the sense that :

(i) It shows that "all" strongly stable boundary conditions are dissipative for an appropriate norm of the solution.

(ii) It gives rise to $\mathrm{L}^{\infty}$-estimates with respect to time instead of $\mathrm{L}^{2}$-estimates.

(iii) These estimates are uniform in time : the constants involved do not depend on time.

2) Such a method, applied to variable coefficients, leads to strong stability results even when these coefficients are not smooth.

This is the point 1) that we shall develop in the part I of this paper. We shall give an illustration of point (2) in the part II.

The outline of this article is as follows. In section 2, we present the basic ideas of our method on the rather simple examples of the second order (section 2.2) and third order (section 2.3) boundary conditions. In each case, we first treat as a model problem the absorbing boundary condition of Engquist-Majda and then extend the result to more general cases. We have chosen to develop this 
section for pedagogical reasons since the ideas for the proof in the general case are not so obvious. Section 3 is devoted to the generalization of results of section 2 to general odd order conditions (section 3.1) and even order conditions (section 3.2).

\section{OBTENTION OF ENERGY TYPE A PRIORI ESTIMATES FOR THE WAVE EQUATION IN THE HALF SPACE : THE CASE OF LOW ORDER CONDITIONS}

As announced in section 1, we consider the wave equation in a $2 \mathrm{D}$ homogeneous medium and consider for simplicity that the propagation velocity is equal to 1 :

$$
\left\{\begin{array}{l}
\frac{\partial^{2} u}{\partial t^{2}}-\Delta u=0 \\
\Delta u=\frac{\partial^{2} u}{\partial x^{2}}+\frac{\partial^{2} u}{\partial y^{2}}
\end{array}\right.
$$

The domain of propagation will be the half-space :

$$
\Omega=\left\{(\mathrm{x}, \mathrm{y}) \in \mathbb{R}^{2}, \mathrm{x} \in \mathbb{R}, \mathrm{y}<0\right\}
$$

and we shall denote by $\Gamma=\partial \Omega$ its boundary. The wave equation will be associated to initial conditions :

$$
\left\{\begin{array}{l}
u(x, y, 0)=u_{0}(x, y) \\
\frac{\partial u}{\partial t}(x, y, 0)=u_{1}(x, y)
\end{array} \quad \text { in } \Omega\right.
$$

which will be the only data of the problem, and to a boundary condition :

$$
\mathrm{Bu}=\mathrm{B}\left(\frac{\partial}{\partial t}, \frac{\partial}{\partial \mathrm{x}}, \frac{\partial}{\partial \mathrm{y}}\right) \mathrm{u}=0 \quad \text { on } \Gamma
$$

where B denotes a homogeneous linear differential operator involving only even order derivatives with respect to the tangential variable $x$.

In this section 2, we shall only consider the cases where $B$ is of order 1,2 or 3 to describe the basic principle of our method. The case of the first order condition is very simple and classical 
(section 2.1). For the second order and third order boundary conditions (sections 2.2 and 2.3) we shall begin by considering the classical absorbing boundary conditions of Engquist-Majda and shall. show that their stability can be proved by energy methods. Then we shall consider the cases of more general second order or third order differential operators and show that the energy method leads exactly to the same conditions than the ones obtained by directly applying the results of HalpernTrefethen. For the case of the second order boundary conditions we shall illustrate on a particular example the difference between strong and weak well-posedness.

The calculations we make in this section are formal. Our goal is to obtain a priori estimates on the solution $u$, if one assumes that this solution exists, is unique and sufficiently regular to justify the technical manipulations we shall be led to do. All this approach can be justified a posteriori by means of functional analysis techniques. In fact almost all our computations will be based on the following well-known identity :

$$
\mid \begin{aligned}
& \text { If } v \text { denotes a sufficiently smooth solution of the wave } \\
& \text { equation } \frac{\partial^{2} v}{\partial t^{2}}-\Delta v=0 \text { in the domain }\{x \in \mathbb{R}, y<0, t>0\} \\
& \text { one has : } \\
& \frac{d}{d t} E(v ; t)=\int_{\Gamma} \frac{\partial v}{\partial y} \frac{\partial v}{\partial t} d x
\end{aligned}
$$

where we have set $\Omega=\{(\mathrm{x}, \mathrm{y}) / \mathrm{x} \in \mathbb{R}, \mathrm{y}<0\}$ and $\Gamma=\partial \Omega$, and where $\mathrm{E}(\mathrm{v} ; \mathrm{t})$ is defined (1.3).

\subsection{The case of the first order boundary condition}

Here we consider the boundary condition

$$
\mathrm{Bu}=\frac{\partial \mathrm{u}}{\partial \mathrm{t}}+\frac{\partial \mathrm{u}}{\partial \mathrm{y}}=0
$$

In this case, it is well-known that, applying (2.4) to the function $u$ itself, leads to the identity :

$$
\frac{d}{d t}\left\{\frac{1}{2} \int_{\Omega}\left|\frac{\partial u}{\partial t}\right|^{2} d x d y+\left.\frac{1}{2} \int_{\Omega}|\nabla u|^{2} d x d y\left|+\int_{\Gamma}\right| \frac{\partial u}{\partial t}\right|^{2} d x=0\right.
$$

which shows that the "first order" energy, which coincides with the physical energy, defined by : 


$$
E_{1}(u ; t)=\frac{1}{2} \int_{\Omega}\left|\frac{\partial u}{\partial t}\right|^{2} d x d y+\frac{1}{2} \int_{\Omega}|\nabla u|^{2} d x d y
$$

is a decreasing function of time. The well-posedness of the initial boundary value problem follows immediately. More precisely one shows that if the energy $E_{1}(0)$ is finite, which means that :

$$
\left(u_{0}, u_{1}\right) \in H^{1}(\Omega) \times L^{2}(\Omega)
$$

then problem (1.2) has a unique weak solution (i.e. in the sense of distributions, see [LM] for instance) which satisfies

$$
\mathrm{u} \in \mathrm{W}^{1, \propto}\left(\mathbb{R}^{+} ; \mathrm{L}^{2}(\Omega)\right) \cap \mathrm{L}^{\alpha}\left(\mathbb{R}^{+} ; \mathrm{H}^{1}(\Omega)\right)
$$

with the estimates

$$
\begin{aligned}
& \left\|\frac{\partial u}{\partial t}\right\|_{L^{-}\left(R^{+} ; L^{2}(\Omega)\right)} \leq C\left(\left\|\nabla u_{0}\right\|_{L^{2}(\Omega)}+\left\|u_{1}\right\|_{L^{2}(\Omega)}\right) \\
& \|\nabla u\|_{L^{2}\left(R^{+} ; L^{2}(\Omega)\right)} \leq C\left(\left\|\nabla u_{0}\right\|_{\left.L^{2}(\Omega)+\left\|u_{1}\right\|_{L^{2}(\Omega)}\right)}\right.
\end{aligned}
$$

Note that such estimates correspond to a strong stability result in the sense of Kreiss since first order derivatives of the solutions can be estimated at a time $t$ by the same derivatives evaluated at the initial time $t=0$; one does not lose any order of derivatives. In fact the result we obtain here is even stronger than the one one gets by a simple and direct application of Kreiss theory since we get here uniform estimates with respect to time (if one uses $\mathrm{L}^{2}$ norms with respect to the space variables) instead of the $-\mathrm{L}^{2}\left(0, \mathrm{~T} ; \mathrm{L}^{2}(\Omega)\right)$ - estimate given by Kreiss theorem. Moreover, as in the theory of Kreiss, we obtain an estimate of the trace of the solution on the boundary $(\Gamma)$ since, thanks to (2.5), we have :

$$
\int_{0}^{+\infty}\left|\frac{\partial u}{\partial t}\right|_{L^{2}(\Gamma)}^{2} d t \leq \frac{1}{2}\left(\left\|u_{1}\right\|_{L^{2}(\Omega)}^{2}+\left\|\nabla u_{0}\right\|_{L^{2}(\Omega)}^{2}\right)
$$

and also, using the boundary condition :

$$
\int_{0}^{+\infty}\left|\frac{\partial u}{\partial y}\right|_{L^{2}(\Gamma)}^{2} d t \leq \frac{1}{2}\left(\left\|u_{1}\right\|_{L^{2}(\Omega)}^{2}+\left\|\nabla_{u_{0}}\right\|_{L^{2}(\Omega)}^{2}\right)
$$




\subsection{The case of second order boundary conditions}

\subsubsection{The classical second order absorbing boundary condition}

We consider now the boundary condition

$$
\mathrm{Bu}=\frac{\partial^{2} \mathrm{u}}{\partial \mathrm{t}^{2}}+\frac{\partial^{2} \mathrm{u}}{\partial \mathrm{y} \partial \mathrm{t}}-\frac{1}{2} \frac{\partial^{2} \mathrm{u}}{\partial \mathrm{x}^{2}}=0
$$

which is known ([EM]) to lead to a strongly well-posed problem. A natural question is : how this well-posedness can appear if one uses energy techniques instead of the normal mode analysis? In fact, it is not clear that, with the condition (2.10), the "first order" energy $E_{1}(u ; t)$ is a decreasing function of time. Nevertheless, we shall see that such a decay occurs for another energy, which will be a "second order" energy in the sense of a positive quadratic form involving second order derivatives of the solution.

To obtain this result we observe that, because of the fact that only the second order derivative in $\mathrm{x}$ occurs in the expression of $\mathrm{Bu}$, the boundary condition (2.10) can be rewritten, as soon as the solution we consider is sufficiently regular, only with derivatives with respect to $y$ and $t$. This was previously observed by Higdon in [Hi 2]. By simply substituting $\frac{\partial^{2} u}{\partial t^{2}}-\frac{\partial^{2} u}{\partial y^{2}}$ to $\frac{\partial^{2} u}{\partial x^{2}}$, we obtain :

$$
\left(\frac{\partial}{\partial t}+\frac{\partial}{\partial y}\right)^{2} u=\frac{\partial^{2} u}{\partial t^{2}}+2 \frac{\partial^{2} u}{\partial t \partial y}+\frac{\partial^{2} u}{\partial y^{2}}=0
$$

that we can also write

$$
\frac{\partial^{2} u}{\partial t^{2}}+\frac{\partial^{2} u}{\partial y^{2}}=-2 \frac{\partial^{2} u}{\partial y \partial t}
$$

Now let us remark that, as we are in the constant coefficient case, if $u$ is a smooth solution of the wave equation, so are $\frac{\partial u}{\partial t}$ and $\frac{\partial u}{\partial y}$. Therefore we can apply our identity (2.4) successively to $v=\frac{\partial u}{\partial t}$ and $v=\frac{\partial u}{\partial y}$ to obtain the two following equalities : 


$$
\mid \begin{array}{ll}
\frac{1}{2} & \frac{d}{d t}\left\{\int_{\Omega}\left(\left|\frac{\partial^{2} u}{\partial t^{2}}\right|^{2}+\left|\frac{\partial^{2} u}{\partial x \partial t}\right|^{2}+\left|\frac{\partial^{2} u}{\partial y \partial t}\right|^{2}\right) d x d y\right\}=\int_{\Gamma} \frac{\partial^{2} u}{\partial t^{2}} \frac{\partial^{2} u}{\partial y \partial t} d x \\
\frac{1}{2} \frac{d}{d t}\left\{\int_{\Omega}\left(\left|\frac{\partial^{2} u}{\partial t \partial y}\right|^{2}+\left|\frac{\partial^{2} u}{\partial x \partial y}\right|^{2}+\left|\frac{\partial^{2} u}{\partial y^{2}}\right|^{2}\right) d x d y\right\}=\int_{\Gamma} \frac{\partial^{2} u}{\partial t \partial y} \frac{\partial^{2} u}{\partial y^{2}} d x
\end{array}
$$

If we now add term by term these two equations it is natural to introduce the following "second order" energy :

$$
E_{2}(u ; t)=\frac{1}{2} \int_{\Omega}\left(\left|\frac{\partial^{2} u}{\partial t^{2}}\right|^{2}+\left|\frac{\partial^{2} u}{\partial x \partial t}\right|^{2}+2\left|\frac{\partial^{2} u}{\partial t \partial y}\right|^{2}+\left|\frac{\partial^{2} u}{\partial x \partial y}\right|^{2}+\left|\frac{\partial^{2} u}{\partial y^{2}}\right|^{2}\right) d x d y
$$

So we have :

$$
\frac{\mathrm{dE}_{2}}{\mathrm{dt}}=\int_{\Gamma}\left(\frac{\partial^{2} \mathrm{u}}{\partial t^{2}}+\frac{\partial^{2} \mathrm{u}}{\partial \mathrm{y}^{2}}\right) \frac{\partial^{2} \mathrm{u}}{\partial \mathrm{y} \partial \mathrm{t}} \mathrm{dx}
$$

that is to say, thanks to $(2.11)$ :

$$
\frac{\mathrm{dE}_{2}}{\mathrm{dt}}=-2 \int_{\Gamma}\left|\frac{\partial^{2} \mathrm{u}}{\partial \mathrm{y} \partial \mathrm{t}}\right|^{2} \mathrm{dx}
$$

which means that the energy $E_{2}(u ; t)$ is a decreasing function of time. In particular it remains finite as soon as :

$$
\left(u_{0}, u_{1}\right) \in H^{2}(\Omega) \times H^{1}(\Omega)
$$

In that case $\mathrm{E}_{2}(\mathrm{u} ; \mathrm{t})$ can be uniformly estimated with the help of $\left\|\mathrm{u}_{0}\right\|_{\mathrm{H}^{2}(\Omega)}^{2}+\left\|\mathrm{u}_{1}\right\|_{\mathrm{H}^{1}(\Omega)}^{2}$. This gives a uniform estimate of all second order derivatives of $u$ but $\frac{\partial^{2} u}{\partial x^{2}}$. But this last estimate follows from the fact that, because of the wave equation, $\frac{\partial^{2} u}{\partial x^{2}}=\frac{\partial^{2} u}{\partial t^{2}}-\frac{\partial^{2} u}{\partial y^{2}}$.

These estimates enable us to prove the existence of a weak solution (in a sense that we shall precise in the next section) of (1.2) satisfying :

$$
\mathrm{u} \in \mathrm{W}^{2,0}\left(0, \mathrm{~T} ; \mathrm{L}^{2}(\Omega)\right) \cap \mathrm{W}^{\mathrm{l}, 0}\left(\mathbb{R}^{+} ; \mathrm{H}^{1}(\Omega)\right) \cap \mathrm{L}^{20}\left(\mathbb{R}^{+} ; \mathrm{H}^{2}(\Omega)\right)
$$

as soon as assumption (2.14) holds. Moreover, the estimate, valid for any second order differential 
operator :

$$
\left\|D^{2} u_{L}\right\|^{-}\left(R^{+} ; L^{2}(\Omega)\right) \leq C\left(\left\|u_{0}\right\|_{H^{2}(\Omega)}+\left\|u_{1}\right\|_{H^{1}(\Omega)}\right)
$$

shows that we have proven a strong stability result (no loss of order of derivatives). Moreover these interior estimates are completed by $\mathrm{L}^{2}$-type estimates on the boundary. Indeed from (2.13), we easily deduce that :

$$
\int_{0}^{+\infty}\left|\frac{\partial^{2} \mathrm{u}}{\partial y \partial t}\right|_{L^{2}(\Gamma)}^{2} \mathrm{dt} \leq \mathrm{C}\left(\left\|\mathrm{u}_{0}\right\|_{\mathrm{H}^{2}(\Omega)}^{2}+\left\|\mathrm{u}_{1}\right\|_{\mathrm{H}^{1}(\Omega)}^{2}\right)
$$

This is not the only boundary estimate one can obtain since for instance (2.10) directly provides an estimate of the quantity $\frac{\partial^{2} \mathrm{u}}{\partial \mathrm{t}^{2}}-\frac{1}{2} \frac{\partial^{2} \mathrm{u}}{\partial \mathrm{x}^{2}}$. Nevertheless both a priori estimates (2.16) and (2.17) are sufficient to give a meaning to $\mathrm{u}$ as a weak solution of problem (1.2). (See the definition (2.25) in the next section 2.2.2).

Note that for the second order boundary condition, we need more regularity on initial data than for the first order condition but we also get more regularity on the solution. Such a fact is not really surprising since the operator $B$ involves higher derivatives which implies more regularity to give a sense to the boundary condition $\mathrm{Bul}_{\Gamma}=0$. One could wonder how to give a sense to a solution of the same problem when we assume only for instance that $\left(u_{0}, u_{1}\right) \in H^{1}(\Omega) \times L^{2}(\Omega)$, which means that only the first order energy $E_{1}(t)$ is finite at $t=0$. This should be possible thanks to a duality process but we shall not examine this point in the present work.

\subsubsection{The case of more general second order boundary conditions}

Let us recall that, in the theory of absorbing boundary conditions for the wave equation ([EM], [TH], [Hi2]), the condition (2.10) corresponds to a Taylor approximation of the function $x \rightarrow \sqrt{1-x^{2}}$ :

$$
\sqrt{1-x^{2}} \simeq 1-\frac{x^{2}}{2}
$$

Of course one can consider approximations of the following type :

$$
\sqrt{1-x^{2}}=\gamma-\beta x^{2} \quad(\beta, \gamma) \in \mathbb{R}^{2}, \beta \neq 0
$$


which leads to the boundary condition :

$$
\gamma \frac{\partial^{2} u}{\partial t^{2}}+\frac{\partial^{2} u}{\partial y \partial t}-\beta \frac{\partial^{2} u}{\partial x^{2}}=0
$$

This is the moment for us to recall the results obtained by Halpern-Trefethen in [TH]. These results concern in particular the well-posedness of the initial boundary value problem obtained by coupling the wave equation to the boundary condition $\mathrm{Bu}=0$ derived from the approximation of the function $\sqrt{1-x^{2}}$ by a general rational function :

$$
\sqrt{1-x^{2}} \simeq R(x)=\frac{P(x)}{Q(x)}
$$

where $P(x), Q(x)$ are polynomials. They give the following

THEOREM A [TH]

The initial boundary value problem (1.2) corresponding to the boundary condition $\mathrm{Bu}=0$ associated with the approximation (2.20) is strongly well-posed if and only if the two following conditions are satisfied :

(i) All the poles and zeros of the rational function $\frac{\mathrm{R}(\mathrm{x})}{\mathrm{X}}$ are real and interlace along the real axis

(ii) $\mathrm{R}(1)>0$ and $\mathrm{R}(-1)>0$.

\section{Remark}

Condition (i) implies in particular that :

$$
0 \leq d^{0} P-d^{0} Q \leq 2
$$

Let us apply this criterion to the condition (2.19) where $R(x)=\gamma-\beta x^{2}$. It is immediate that (i) holds if and only if $\gamma \beta>0$ while criterion (ii) implies $\gamma-\beta>0$. Therefore, problem (1.2) associated to (2.19) is strongly well-posed if and only if :

$$
0<\beta<\gamma
$$

Let us see now how this condition appears when one uses energy techniques. Of course we still can use the two equalities (2.12) which are independent on the boundary condition. Now instead of summing term by term these two equalities we first multiply the first of them by a the second one by $(\gamma-\beta)$ and add the two results. We obtain : 


$$
\frac{d E_{2}}{d t}=+\int_{\Gamma}\left((\gamma-\beta) \frac{\partial^{2} u}{\partial t^{2}}+\beta \frac{\partial^{2} u}{\partial y^{2}}\right) \frac{\partial^{2} u}{\partial y \partial t} d x
$$

where the function $E_{2}(u ; t)$ is defined by

$$
\mid \begin{aligned}
E_{2}(u ; t) & =\frac{\gamma-\beta}{2} \int_{\Omega}\left(\left|\frac{\partial^{2} u}{\partial t^{2}}\right|^{2}+\left|\frac{\partial^{2} u}{\partial x \partial t}\right|^{2}+\left|\frac{\partial^{2} u}{\partial y \partial t}\right|^{2}\right) d x d y \\
& +\frac{\beta}{2} \int_{\Omega}\left(\left|\frac{\partial^{2} u}{\partial y \partial t}\right|^{2}+\left|\frac{\partial^{2} u}{\partial x \partial y}\right|^{2}+\left|\frac{\partial^{2} u}{\partial y^{2}}\right|^{2}\right) d x d y
\end{aligned}
$$

Now we observe that, replacing $\frac{\partial^{2} u}{\partial x^{2}}$ by $\frac{\partial^{2} u}{\partial t^{2}}-\frac{\partial^{2} u}{\partial y^{2}}$, the boundary condition (2.19) is equivalent to :

$$
(\gamma-\beta) \frac{\partial^{2} u}{\partial t^{2}}+\beta \frac{\partial^{2} u}{\partial y^{2}}=-\frac{\partial^{2} u}{\partial y \partial t}
$$

so that $(2.22)$ becomes :

$$
\frac{\mathrm{dE}_{2}}{\mathrm{dt}}=-\int_{\Gamma}\left|\frac{\partial^{2} \mathrm{u}}{\partial \mathrm{y} \partial \mathrm{t}}\right|^{2} \mathrm{dx}
$$

This proves that the function $E_{2}(u ; t)$ is decaying in time. To be able to deduce from this fact a priori estimates on the solution, we need that $E_{2}(u ; t)$ be a "second order energy", i.e. a positive quadratic form. This implies $0 \leq \beta \leq \gamma$. Now if we want to estimate all the second order derivatives of $u$, which is necessary to get the strong stability property, we see that we must have :

$$
0<\beta<\gamma
$$

that is to say exactly the condition (2.21). If (2.21) holds it is then easy, mimicking what we did in section 2.2.1, to obtain the estimates (2.16) and (2.17) and then to prove the existence of a unique weak solution of $u$. This must be understood in the following sense : 


$$
\mid \begin{aligned}
& \cdot u(x, y, t) \text { satisfies }(2.15) \\
& \cdot \forall v(x, y) \in V=\left\{v \in H^{1}(\Omega) /\left.v\right|_{\Gamma} \in H^{1}(\Gamma)\right\} \\
& \quad \frac{d^{3}}{d^{3}}\left(\int_{\Omega} u v d x d y\right)+\gamma \frac{d^{2}}{d^{2}}\left(\int_{\Gamma} u v d x\right) \\
& \quad+\frac{d}{d t}\left(\int_{\Omega} \nabla u . \nabla v d x d y\right)+\beta \int_{\Gamma} \frac{\partial u}{\partial x} \frac{\partial v}{\partial x} d x=0 \quad \text { in } D^{\prime}(0, T) \\
& \cdot u(x, y, 0)=u_{d}(x, y) ; \frac{\partial u}{\partial t}(x, y, 0)=u_{1}(x, y)
\end{aligned}
$$

Let us state our result in the following theorem:

\section{THEOREM 2.1}

Under the condition (2.21), the initial boundary value problem $((2.1),(2.19))$ is strongly well-posed in the sense of Kreiss and the unique solution satisfies the identity (2.24) which yields the decay in time of the second order energy $E_{2}$ defined by (2.23). Moreover, this solution satisfies the a priori estimate:

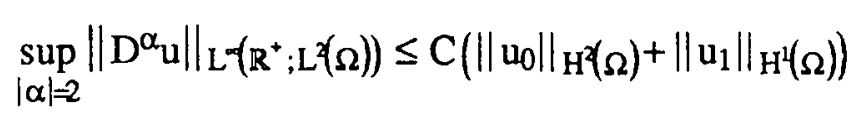

where the positive constant $C$ only depends on $\gamma$ and $\beta$.

\section{Remark}

If one does not look for a strong stability result in the sense of Kreiss but only a well-posedness result in the sense of Hadamard, it is sufficient to apply the condition of Hersch [He]. In our particular case, this condition implies simply that :

$$
\gamma>0, \beta>0
$$

This means that for $0<\gamma<\beta<+\infty$, problem (1.2) is only weakly well-posed. Such a fact can also be obtained by using energy techniques in order to get a priori estimates. Indeed let $u$ be a regular solution of (1.2), let us introduce :

$$
v=\gamma \frac{\partial^{2} u}{\partial t^{2}}+\frac{\partial^{2} u}{\partial y \partial t}-\beta \frac{\partial^{2} u}{\partial x^{2}}
$$

It is clear that $\mathrm{v}$ is solution of : 


$$
\begin{cases}\frac{\partial^{2} v}{\partial t^{2}}-\Delta v=0 & \text { in } \Omega \\ \left.v\right|_{\Gamma}=0 & \text { on } \Gamma\end{cases}
$$

which is a well-posed problem. Now if we want to get estimates on u we have only to check that the initial value problem:

$$
\begin{aligned}
& \gamma \frac{\partial^{2} \mathrm{u}}{\partial \mathrm{t}^{2}}+\frac{\partial^{2} \mathrm{u}}{\partial \mathrm{y} \partial \mathrm{t}}-\beta \frac{\partial^{2} \mathrm{u}}{\partial \mathrm{x}^{2}}=\mathrm{v} \quad(\mathrm{x}, \mathrm{y}) \in \Omega, \mathrm{t}>0 \\
& \left\{\begin{array}{l}
u(x, 0)=u_{0}(x, y) \\
\frac{\partial u}{\partial t}(x, 0)=u_{1}(x, y)
\end{array}\right.
\end{aligned}
$$

is well-posed. But multiplying equation (2.27) by $\frac{\partial u}{\partial t}$ and integrating over $\Omega$ gives :

$$
\mid \begin{aligned}
& \frac{d}{d t}\left\{\frac{\gamma}{2} \int_{\Omega}\left|\frac{\partial u}{\partial t}\right|^{2} d x d y+\frac{\beta}{2} \int_{\Omega}\left|\frac{\partial u}{\partial x}\right|^{2} d x d y \mid\right. \\
& +\int_{\Gamma}\left|\frac{\partial u}{\partial t}\right|^{2} d x=\int_{\Omega} v \frac{\partial u}{\partial t} d x d y
\end{aligned}
$$

which easily leads to the following estimates in the interval $[0, T]$ (use Gronwall's Lemma) :

$$
\| \begin{aligned}
& \left\|\frac{\partial u}{\partial t}\right\|_{L^{-}\left(0, T ; L^{2}\right)} \leq C(T)\|v\|_{L^{2}\left(0, T ; L^{2}\right)} \\
& \left\|\frac{\partial u}{\partial x}\right\|_{L-\left(0, T ; L^{2}\right)} \leq C(T)\|v\|_{L^{2}\left(0, T ; L^{2}\right)}
\end{aligned}
$$

Moreover, multiplying equation (2.27) by $\frac{\partial u}{\partial t}+\frac{\partial u}{\partial y}$ and integrating over $\Omega$, we get :

$$
\left.\mid \begin{array}{l}
\frac{d}{d t}\left\{\frac{\gamma}{2} \int_{\Omega}\left|\frac{\partial u}{\partial t}+\frac{\partial u}{\partial y}\right|^{2} d x d y+\frac{\beta}{2} \int_{\Omega}\left|\frac{\partial u}{\partial x}\right|^{2} d x d y\right.
\end{array}\right\}
$$

which leads to the estimate :

$$
\left.\left\|\frac{\partial u}{\partial y}\right\|_{L \cdot\left(0, T ; L^{2}\right)} \leq \mathrm{CT}\right)\|v\|_{L^{2}\left(0, T ; L^{2}\right)}
$$


We need now an estimate about $\mathrm{v}$ solution of (2.27). This problem can be solved explicitly using the theory of images for the Dirichlet condition. Then, by a Fourier transform in space, one easily checks that :

$$
\|v(t)\|_{L^{2}(\Omega)}^{2} \leq 2\left\{\left\|v_{0}\right\|_{L^{2}(\Omega)}^{2}+\left\|v_{1}\right\|_{H^{-1}(\Omega)}^{2}\right\}
$$

where $v(x, y, 0)=v_{0}(x, y)$ and $\frac{\partial v}{\partial t}(x, y, 0)=v_{1}(x, y)$. But, thanks to the definition of $v$, we have :

$$
\left\{\begin{array}{l}
v_{0}=\gamma \frac{\partial^{2} u_{0}}{\partial x^{2}}+(\gamma-\beta) \frac{\partial^{2} u_{0}}{\partial y^{2}}+\frac{\partial u_{1}}{\partial y} \\
v_{1}=\frac{\partial^{2} u_{1}}{\partial x^{2}}+(\gamma-\beta) \frac{\partial^{2} u_{1}}{\partial y^{2}}+\frac{\partial}{\partial y} \Delta u_{0}
\end{array}\right.
$$

so that :

$$
\left\|v_{0}\right\|_{L^{2}(\Omega)}^{2}+\left\|v_{1}\right\|_{H^{-1}(\Omega)}^{2} \leq C\left\{\left\|u_{0}\right\|_{H^{x}(\Omega)}^{2}+\left\|u_{1}\right\|_{H^{1}(\Omega)}^{2}\right\}
$$

Regrouping (2.29), (2.30), (2.31) and (2.32), we obtain :

$$
\|\mathrm{Du}\|_{\mathrm{L}-\left(0, \mathrm{~T} ; \mathrm{L}^{2}\right)} \leq \mathrm{C}(\mathrm{T})\left\{\left\|\mathrm{u}_{0}\right\|_{\mathrm{H}^{2}(\Omega)}+\left\|\mathrm{u}_{1}\right\|_{\mathrm{H}^{1}(\Omega)}\right\}
$$

This proves the well-posedness of the initial boundary problem as soon as as $\beta, \gamma>0$. Nevertheless this result corresponds only to a weak stability result since we lose one order of derivative in our estimates.

\subsection{The case of third order boundary conditions}

\subsubsection{The classical third order absorbing boundary condition}

We consider now the classical third order condition :

$$
\frac{\partial^{3} u}{\partial t^{3}}+\frac{\partial^{3} u}{\partial t^{2} \partial y}-\frac{3}{4} \frac{\partial^{3} u}{\partial t \partial x^{2}}-\frac{1}{4} \frac{\partial^{3} u}{\partial x^{2} \partial y}=0
$$

which is known [Hi2], at least for smooth solutions, to be equivalent to : 


$$
\left(\frac{\partial}{\partial t}+\frac{\partial}{\partial y}\right)^{3} u=0
$$

that we can also write :

$$
\frac{\partial^{3} u}{\partial t^{3}}+\frac{\partial^{3} u}{\partial t^{3} \partial y}+\frac{\partial^{3} u}{\partial t \partial y^{2}}+\frac{\partial^{3} u}{\partial y^{3}}=-2\left(\frac{\partial^{3} u}{\partial t^{2} \partial y}+\frac{\partial^{3} u}{\partial t \partial y^{2}}\right)
$$

The idea is now to apply the identity (2.4) to the two functions :

$$
\mid \begin{aligned}
& v_{1}=\frac{\partial^{2} u}{\partial t^{2}}+\frac{\partial^{2} u}{\partial t \partial y} \\
& v_{2}=\frac{\partial^{2} u}{\partial t \partial y}+\frac{\partial^{2} u}{\partial y^{2}}
\end{aligned}
$$

which are particular solutions of the wave equation as soon as $u$ is also a solution. Moreover, they have the property that $\frac{\partial \mathrm{v}_{1}}{\partial \mathrm{y}}=\frac{\partial \mathrm{v}_{2}}{\partial \mathrm{t}}$. We thus have :

$$
\mid \begin{aligned}
& \frac{d}{d t} E\left(v_{1}\right)=\int_{\Gamma}\left(\frac{\partial^{3} u}{\partial t^{3}}+\frac{\partial^{3} u}{\partial t^{2} \partial y}\right)\left(\frac{\partial^{3} u}{\partial t^{2} \partial y}+\frac{\partial^{3} u}{\partial t \partial y^{2}}\right) d x \\
& \frac{d}{d t} E\left(v_{2}\right)=\int_{\Gamma}\left(\frac{\partial^{3} u}{\partial t^{2} \partial y}+\frac{\partial^{3} u}{\partial t \partial y^{2}}\right)\left(\frac{\partial^{3} u}{\partial t \partial y^{2}}+\frac{\partial^{3} u}{\partial y^{3}}\right) d x
\end{aligned}
$$

Summing these two equalities leads to :

$$
\frac{d}{d t}\left\{E\left(v_{1}\right)+E\left(v_{2}\right)\right\}=\int_{\Gamma}\left(\frac{\partial^{3} u}{\partial t^{2} \partial y}+\frac{\partial^{3} u}{\partial t \partial y^{2}}\right)\left(\frac{\partial^{3} u}{\partial t^{3}}+\frac{\partial^{3} u}{\partial t^{2} \partial y}+\frac{\partial^{3} u}{\partial t \partial y^{2}}+\frac{\partial^{3} u}{\partial y^{3}}\right) d x
$$

that is to say, thanks to $(2.35)$ :

$$
\frac{d}{d t}\left\{E\left(v_{1}\right)+E\left(v_{2}\right)\right\}=-2 \int_{\Gamma}\left|\frac{\partial^{3} u}{\partial t^{2} \partial y}+\frac{\partial^{3} u}{\partial t \partial y^{2}}\right|^{2} d x
$$

which means that the quadratic form : 


$$
\begin{aligned}
E\left(v_{1}\right)+E\left(v_{2}\right) & =\frac{1}{2} \int_{\Omega}\left(\left|\frac{\partial^{3} u}{\partial t^{3}}+\frac{\partial^{3} u}{\partial t^{2} \partial y}\right|^{2}+\left|\frac{\partial^{3} u}{\partial t^{2} \partial x}+\frac{\partial^{3} u}{\partial x \partial y \partial t}\right|^{2}+\left|\frac{\partial^{3} u}{\partial t^{2} \partial y}+\frac{\partial^{3} u}{\partial t \partial y^{2}}\right|^{2}\right) d x d y \\
& +\frac{1}{2} \int_{\Omega}\left(\left|\frac{\partial^{3} u}{\partial t^{2} \partial y}+\frac{\partial^{3} u}{\partial t \partial y^{2}}\right|^{2}+\left|\frac{\partial^{3} u}{\partial t \partial x \partial y}+\frac{\partial^{3} u}{\partial y^{2} \partial x}\right|^{2}+\left|\frac{\partial^{3} u}{\partial t \partial y^{2}}+\frac{\partial^{3} u}{\partial y^{3}}\right|^{2}\right) d x d y
\end{aligned}
$$

is a decreasing function of time. Nevertheless, the estimates we can deduce from this fact do not permit us to conclude to a strong stability result since we do not control all the third order derivatives of $\mathrm{u}$ but only some linear combinations of them. Therefore we have to go into the second step of our estimates which consists in applying the identity (2.4) to the function $v=\frac{\partial^{2} u}{\partial t \partial y}$. By this process, we obtain :

$$
\frac{d}{d t}\left\{\frac{1}{2} \int_{\Omega}\left(\left|\frac{\partial^{3} u}{\partial t^{2} \partial y}\right|^{2}+\left|\frac{\partial^{3} u}{\partial t \partial x \partial y}\right|^{2}+\left|\frac{\partial^{3} u}{\partial t \partial y}\right|^{2}\right) d x d y\right\}=\int_{\Gamma} \frac{\partial^{3} u}{\partial t \partial y^{2}} \frac{\partial^{3} u}{\partial y \partial t^{2}} d x
$$

Noticing that :

$$
2(a+b)^{2}-a b=\frac{3}{2}(a+b)^{2}+\frac{1}{2}\left(a^{2}+b^{2}\right)
$$

we see that, if we introduce the third order energy

$$
\mid \begin{aligned}
& E_{3}(u)=E(v)+E\left(v_{1}\right)+E\left(v_{2}\right) \\
& E(v)=\frac{1}{2} \int_{\Omega}\left[\left|\frac{\partial^{3} u}{\partial t^{2} \partial y}\right|^{2}+\left|\frac{\partial^{3} u}{\partial t \partial x \partial y}\right|^{2}+\left|\frac{\partial^{3} u}{\partial t \partial y^{2}}\right|^{2}\right] d x d y \\
& E\left(v_{1}\right)=\frac{1}{2} \int\left[\left|\frac{\partial^{3} u}{\partial t^{3}}+\frac{\partial^{3} u}{\partial t^{2} \partial y}\right|^{2}+\left|\frac{\partial^{3} u}{\partial t^{2} \partial x}+\frac{\partial^{3} u}{\partial t \partial x \partial y}\right|^{2}+\left|\frac{\partial^{3} u}{\partial t^{2} \partial y}+\frac{\partial^{3} u}{\partial t \partial y^{2}}\right|^{2}\right] d x d y \\
& E\left(v_{2}\right)=\frac{1}{2} \int\left[\left|\frac{\partial^{3} u}{\partial t^{2} \partial y}+\frac{\partial^{3} u}{\partial t \partial y^{2}}\right|^{2}+\left|\frac{\partial^{3} u}{\partial t \partial x \partial y}+\frac{\partial^{3} u}{\partial x \partial y^{2}}\right|^{2}+\left|\frac{\partial^{3} u}{\partial t \partial y^{2}}+\frac{\partial^{3} u}{\partial y^{3}}\right|^{2}\right] d x d y
\end{aligned}
$$

the addition of (2.37) and (2.38) leads to :

$$
\frac{d E_{3}}{d t}(u ; t)+\frac{3}{2} \int_{\Gamma}\left|\frac{\partial^{3} u}{\partial t \partial y^{2}}+\frac{\partial^{3} u}{\partial t^{2} \partial y}\right|^{2} d x+\frac{1}{2} \int_{\Gamma}\left(\left|\frac{\partial^{3} u}{\partial t \partial y^{2}}\right|^{2}+\left|\frac{\partial^{3} u}{\partial t^{2} \partial y}\right|^{2}\right) d x=0
$$

which proves that the energy $E_{3}(u ; t)$ decays in time. In particular if it is finite a $t=0$, i.e. if one has 


$$
\left(\mathrm{u}_{0}, \mathrm{u}_{1}\right) \in \mathrm{H}^{3}(\Omega) \times \mathrm{H}^{2}(\Omega)
$$

it remains uniformly bounded in time. From (2.40), we easily see that we get a priori estimates of the $\mathrm{L}^{2}$-norms of all the third order derivatives of $\mathrm{u}$ except the ones containing second or third order derivatives with respect to $x$. Anyway, analogous estimates for these quantities can be derived from the wave equation that we can differentiate in $t, x$ and $y$ to obtain :

$$
\left\{\begin{array}{l}
\frac{\partial^{3} u}{\partial t \partial x^{2}}=\frac{\partial^{3} u}{\partial t^{3}}-\frac{\partial^{3} u}{\partial t \partial y^{2}} \\
\frac{\partial^{3} u}{\partial y \partial x^{2}}=\frac{\partial^{3} u}{\partial t^{2} \partial y}-\frac{\partial^{3} u}{\partial y^{3}} \\
\frac{\partial^{3} u}{\partial x^{3}}=\frac{\partial^{3} u}{\partial t^{2} \partial x}-\frac{\partial^{3} u}{\partial x \partial y^{2}}
\end{array}\right.
$$

As these estimates are uniform in time, we can write for any linear third order differential operator :

$$
\left\|D^{3} u\right\|_{L^{-}\left(\mathbb{R}^{+} ; L^{2}(\Omega)\right)} \leq \mathrm{C}\left(\left\|u_{0}\right\|_{H^{3}}+\left\|u_{1}\right\|_{H^{2}}\right)
$$

which establishes the strong stability of the initial boundary value problem (1.2) when one uses the boundary condition (2.34).

Of course, as in the second order case, we get $L^{2}$-estimates on the boundary $\Gamma$ since from (2.39) we directly obtain that :

$$
\int_{0}^{+\infty} \int_{\Gamma}\left(\left|\frac{\partial^{3} u}{\partial t \partial y^{2}}\right|^{2}+\left|\frac{\partial^{3} u}{\partial t^{2} \partial y}\right|^{2}\right) d x d t \leq C\left\{\left\|u_{0}\right\|_{\mathrm{H}^{3}}^{2}+\left\|u_{1}\right\|_{\mathrm{H}^{2}}^{2}\right\}
$$

which is the equivalent of the estimate (2.15) we obtained in the second order case.

\subsubsection{The case of general third order boundary conditions}

Condition (2.34) follows from the following Padé approximation of the function $\sqrt{1-x^{2}}$ 


$$
\sqrt{1-x^{2}}=1-\frac{\frac{1}{2} x^{2}}{1-\frac{1}{4} x^{2}}
$$

Let us generalize this condition by considering the following class of rational approximations of $\sqrt{1-x^{2}}$ :

$$
\sqrt{1-x^{2}} \simeq \gamma-\frac{\beta x^{2}}{1-\alpha x^{2}} \quad(\alpha, \beta, \gamma) \in \mathbb{R}^{3}
$$

which leads to the general third order condition :

$$
\gamma \frac{\partial^{3} u}{\partial t^{3}}+\frac{\partial^{3} u}{\partial t^{2} \partial y}-(\alpha+\beta) \frac{\partial^{3} u}{\partial x^{2} \partial t}-\alpha \frac{\partial^{3} u}{\partial x^{2} \partial y}=0
$$

the stability of which we wish to analyze using energy techniques. For the sake of simplicity, we shall restrict ourselves to the particular case $\gamma=1$. However, all what we shall do can be extended without any supplementary difficulty (except purely technical difficulty) and this assumption will simply allows us to shorten this section. We thus consider the boundary condition :

$$
\left\{\begin{array}{l}
\frac{\partial^{3} u}{\partial t^{3}}+\frac{\partial^{3} u}{\partial t^{2} \partial y}-(\alpha+\beta) \frac{\partial^{3} u}{\partial x^{2} \partial t}-\alpha \frac{\partial^{3} u}{\partial x^{2} \partial y}=0 \\
(\alpha, \beta) \in \mathbb{R}^{*} \times \mathbb{R}^{*}
\end{array}\right.
$$

If we refer to theorem 1 to analyse the strong stability of (2.47) it is easy to see that, for $\mathrm{R}(\mathrm{x})=1-\frac{\beta \mathrm{x}^{2}}{1-\alpha \mathrm{x}^{2}}$, we have :

(i) holds $\Leftrightarrow \alpha>0, \beta>\alpha$

(ii) holds $\Leftrightarrow \beta(1-\alpha)>0$.

Therefore, the necessary and sufficient condition for problem (1.2) to be strongly well-posed is:

$$
\alpha>0, \beta>0, \quad 0<\alpha+\beta<1
$$

Our purpose in this section is now to show how these conditions naturally arise when one tries to obtain energy estimates using a method analogous to the one we have used in section 2.2.1.

Since the computations are rather lengthy, let us decompose this approach in several steps. 
First step :

Let us consider a real number $a$ and the two following functions :

$$
\left\{\begin{array}{l}
v_{1}=\frac{\partial^{2} u}{\partial t^{2}}+a \frac{\partial^{2} u}{\partial t \partial y} \\
v_{2}=\frac{\partial^{2} u}{\partial t \partial y}+a \frac{\partial^{2} u}{\partial y^{2}}
\end{array}\right.
$$

u denoting a (sufficiently smooth) function satisfying the wave equation in $\Omega$ and the boundary condition (2.47) on $(\Gamma)$. Note that $v_{1}$ and $v_{2}$ are derivatives with respect to $t$ and $y$ of the same function $\frac{\partial u}{\partial t}+a \frac{\partial u}{\partial y}$ and are thus related by $\frac{\partial v_{1}}{\partial y}=\frac{\partial v_{2}}{\partial t}$. Applying identity (2.4), we obtain :

$$
\mid \begin{aligned}
& \frac{d}{d t}\left\{E\left(v_{1}\right)\right\}=\int_{\Gamma}\left(\frac{\partial^{3} u}{\partial t^{3}}+a \frac{\partial^{3} u}{\partial t^{2} \partial y}\right)\left(\frac{\partial^{3} u}{\partial t^{2} \partial y}+a \frac{\partial^{3} u}{\partial t \partial y^{2}}\right) d x \\
& \frac{d}{d t}\left\{E\left(v_{2}\right)\right\}=\int_{\Gamma}\left(\frac{\partial^{3} u}{\partial t^{2} \partial y}+a \frac{\partial^{3} u}{\partial t \partial y^{2}}\right)\left(\frac{\partial^{3} u}{\partial t \partial y^{2}}+a \frac{\partial^{3} u}{\partial y^{3}}\right) d x
\end{aligned}
$$

So if $\mathrm{b}$ denotes a real number, we have :

$$
\frac{d}{d t}\left\{b E\left(v_{1}\right)+E\left(v_{2}\right)\right\}=\int_{\Gamma}\left(\frac{\partial^{3} u}{\partial t^{2} \partial y}+a \frac{\partial^{3} u}{\partial t \partial y^{2}}\right)\left(b \frac{\partial^{3} u}{\partial t^{3}}+a b \frac{\partial^{3} u}{\partial t^{2} \partial y}+\frac{\partial^{3} u}{\partial t \partial y^{2}}+a \frac{\partial^{3} u}{\partial y^{3}}\right) d x
$$

Second step :

We eliminate the $\mathrm{x}$ derivatives in the boundary condition (2.47) that we rewrite :

$$
[1-(\alpha+\beta)] \frac{\partial^{3} u}{\partial t^{3}}+(1-\alpha) \frac{\partial^{3} u}{\partial t^{2} \partial y}+(\alpha+\beta) \frac{\partial^{3} u}{\partial t \partial y^{2}}+\alpha \frac{\partial^{3} u}{\partial y^{3}}=0
$$

We now choose :

$$
\left\{\begin{array}{l}
a=\lambda \alpha \\
b=\lambda(1-\alpha-\beta)
\end{array}\right.
$$

so that, thanks to $(2.52)$ : 


$$
a \frac{\partial^{3} u}{\partial y^{3}}+b \frac{\partial^{3} u}{\partial t^{3}}=-\lambda\left\{(1-\alpha) \frac{\partial^{3} u}{\partial t^{2} \partial y}+(\alpha+\beta) \frac{\partial^{3} u}{\partial y^{2} \partial y}\right\}
$$

Thus, identity (2.51) leads to

$$
\mid \begin{aligned}
& \frac{d}{d t}\left\{b E\left(v_{1}\right)+E\left(v_{2}\right)\right)= \\
& =\int_{\Gamma}\left(\frac{\partial^{3} u}{\partial y \partial t^{2}}+\lambda \alpha \frac{\partial^{3} u}{\partial y^{2} \partial t}\right)\left(\left[\lambda^{2} \alpha(1-\alpha-\beta)-\lambda(1-\alpha)\right] \frac{\partial^{3} u}{\partial y \partial t^{2}}+[1-\lambda(\alpha+\beta)] \frac{\partial^{3} u}{\partial y^{2} \partial t}\right) d x
\end{aligned}
$$

Let us choose $\lambda$ in order that :

$$
\lambda \alpha\left[\lambda^{2} \alpha(1-\alpha-\beta)-\lambda(1-\alpha)\right]=1-\lambda(\alpha+\beta)
$$

which means that $\lambda$ is one the roots of the following polynomial equation :

$$
P(\lambda)=\lambda^{3} \alpha^{2}(1-\alpha-\beta)-\lambda^{2} \alpha(1-\alpha)+\lambda(\alpha+\beta)-1=0
$$

In that case, we have :

$$
\frac{d}{d t}\left\{E\left(v_{2}\right)+b E\left(v_{1}\right)\right\}=\left[\lambda^{2} \alpha(1-\alpha-\beta)-\lambda(1-\alpha)\right] \int_{\Gamma}\left|\frac{\partial^{3} u}{\partial y \partial t^{2}}+\lambda \alpha \frac{\partial^{3} u}{\partial y^{2} \partial t}\right|^{2} d x
$$

To deduce appropriate a priori estimates on $v_{1}$ and $v_{2}$ from (2.55), we have to satisfy:

$$
\left\{\begin{array}{l}
b=\lambda(1-\alpha-\beta)>0 \\
\lambda^{2} \alpha(1-\alpha-\beta)-\lambda(1-\alpha) \leq 0
\end{array}\right.
$$

Nevertheless this is not sufficient to conclude to a strong stability result since the fact that $E\left(v_{1}\right)$ and $E\left(v_{2}\right)$ is bounded does not lead to uniform estimates of all the third order derivatives of $u$ in the domain $\Omega$. To overcome this difficulty we also apply our basic identity (2.4) to the function $v_{3}=\frac{\partial^{2} u}{\partial y \partial t}$, exactly as we did in section 2.3.1: 


$$
\frac{\mathrm{d}}{\mathrm{dt}}\left\{\mathrm{E}\left(\frac{\partial^{2} \mathrm{u}}{\partial \mathrm{y} \partial \mathrm{t}}\right)\right\}=\int_{\Gamma} \frac{\partial^{2} \mathrm{u}}{\partial \mathrm{y} \partial \mathrm{t}^{2}} \cdot \frac{\partial^{2} \mathrm{u}}{\partial \mathrm{y}^{2} \partial \mathrm{t}} \mathrm{dx}
$$

We multiply (2.57) by some $\delta>0$ and add the result to (2.55) which gives :

$$
\mid \begin{aligned}
& \frac{d}{d t}\left\langle b E\left(v_{1}\right)+E\left(v_{2}\right)+\delta E\left(\frac{\partial^{2} u}{\partial y \partial t}\right)\right)= \\
& =\left[\lambda^{2} \alpha(1-\alpha-\beta)-\lambda(1-\alpha)\right] \int_{\Gamma}\left|\frac{\partial^{3} u}{\partial y \partial t^{2}}+\lambda \alpha \frac{\partial^{3} u}{\partial y^{2} \partial t}\right|^{2} d x \\
& +\delta \int_{\Gamma} \frac{\partial^{3} u}{\partial y^{2} \partial t} \cdot \frac{\partial^{3} u}{\partial y \partial t^{2}} d x
\end{aligned}
$$

In order that the right hand side member of (2.58) be a negative quadratic form, we need of course that $\lambda^{2} \alpha(1-\alpha-\beta)-\lambda(1-\alpha) \leq 0$ but this is not sufficient. Indeed, let us set :

$$
\left\{\begin{array}{l}
\delta=\left\{\lambda^{2} \alpha(1-\alpha-\beta)-\lambda(1-\alpha)\right\} \delta^{\prime} \\
E_{3}(u)=\delta E\left(\frac{\partial^{2} u}{\partial y \partial t}\right)+E\left(v_{2}\right)+b E\left(v_{1}\right)
\end{array}\right.
$$

we have

$$
\frac{d}{d t} E_{3}(u)=\left\{\lambda^{2} \alpha(1-\alpha-\beta)-\lambda(1-\alpha)\right\} \int_{\Gamma}\left\{\left|\frac{\partial^{3} u}{\partial y \partial t^{2}}+\lambda \alpha \frac{\partial^{3} u}{\partial y^{2} \partial t}\right|^{2}+\delta^{\prime} \frac{\partial^{3} u}{\partial y^{2} \partial t} \frac{\partial^{3} u}{\partial y \partial t^{2}}\right\} d x
$$

To be able to conclude, we must find $\delta^{\prime}<0$ such that the quadratic form $(x+\lambda \alpha y)^{2}+\delta^{\prime} x y$ is positive. As the discriminant of this quadratic form is :

$$
\Delta=\delta^{\prime 2}-\lambda \alpha
$$

We simply have to ensure that $\Delta<0$. This implies :

$$
\lambda \alpha>0
$$

in which case it suffices to take :

$$
\delta^{\prime}=-\frac{1}{2} \sqrt{\lambda \alpha}
$$


and the coefficient $\delta$ will be strictly positive as soons as :

$$
\lambda^{2} \alpha(1-\alpha-\beta)-\lambda(1-\alpha)<0
$$

Let us now regroup all our conditions. We see that if one can find a real number $\lambda$ such that :

$$
\left\{\begin{array}{l}
\mathrm{P}(\lambda)=\lambda^{3} \alpha^{2}(1-\alpha-\beta)-\lambda^{2} \alpha(1-\alpha)+\lambda(\alpha+\beta)-1=0 \\
\lambda^{2} \alpha(1-\alpha-\beta)-\lambda(1-\alpha)<0 \\
\lambda(1-\alpha-\beta)>0 \\
\lambda \alpha>0
\end{array}\right.
$$

then, the third order energy :

$$
E_{3}(u)=b E\left(v_{1}\right)+E\left(v_{2}\right)+\delta E\left(\frac{\partial^{2} u}{\partial y \partial t}\right)
$$

where $(b, \delta)$ are strictly positive numbers, is a decreasing function of time and is thus uniformly bounded in time as soon as :

$$
\left(u_{0}, u_{1}\right) \in H^{3}(\Omega) \times H^{2}(\Omega)
$$

This leads to uniform estimates in time of the $\mathrm{L}^{2}$-norms in space of all the third order derivatives of $u$ but $\frac{\partial^{3} u}{\partial t \partial x^{2}}, \frac{\partial^{3} u}{\partial x^{2} \partial y}, \frac{\partial^{3} u}{\partial x^{3}}$. Nevertheless these quantities are easily estimated using the wave equation as in section 2.3.1. Finally, as soon as we can find $\lambda$ solution of (2.63), we get the following interior estimates :

$$
\left\|D^{3} u\right\|_{L}\left(R^{+} ; L^{2}(\Omega)\right) \leq C\left\{\left\|u_{0}\right\|_{H^{3}(\Omega)}+\left\|u_{1}\right\|_{H^{2}(\Omega)}\right\}
$$

that we can complete with boundary estimates, as we have seen in section 2.3.1.

$$
\left\{\begin{array}{l}
\left\|\frac{\partial^{3} u}{\partial y \partial t^{2}}\right\|_{L^{2}\left(\mathbb{R}^{+} ; L^{2}(\Gamma)\right)} \leq C\left\{\left\|u_{0}\right\|_{H^{3}(\Omega)}+\left\|u_{1}\right\|_{H^{2}(\Omega)}\right\} \\
\left\|\frac{\partial^{3} u}{\partial y^{2} \partial t}\right\|_{L^{2}\left(\mathbb{R}^{+} ; L^{2}(\Gamma)\right)} \leq C\left\{\left\|u_{0}\right\|_{H^{3}(\Omega)}+\left\|u_{1}\right\|_{H^{2}(\Omega)}\right\}
\end{array}\right.
$$

It remains to see what are the conditions on $\mathrm{a}$ and $\mathrm{b}$ in order that one can find some $\lambda$ satisfying (2.63). For this let us introduce : 


$$
x=\lambda \alpha
$$

so that the system (2.63) becomes :

$$
\left\{\begin{array}{l}
F(x)=[1-(\alpha+\beta)] x^{3}-(1-\alpha) x^{2}-(\alpha+\beta) x-\alpha=0 \\
x>0 \\
\alpha x(1-(\alpha+\beta))>0 \\
\alpha x^{2}(1-(\alpha+\beta))-\alpha x(1-\alpha)<0
\end{array}\right.
$$

The two first inequalities imply that :

$$
x^{2}(1-(\alpha+\beta))>0
$$

Suppose that $\alpha<0$, then $1-\alpha>1$ and $\alpha x^{2}(1-(\alpha+\beta))-\alpha x(1-\alpha)>0$, which is impossible because of the third inequality of (2.69). Thus $\alpha$ is strictly positive and the first two inequalities of (2.69) are equivalent to :

$$
\left\{\begin{array}{l}
x>0 \\
\alpha+\beta<1
\end{array}\right.
$$

Moreover the equation $\mathrm{F}(\mathrm{x})=0$ can be rewritten

$$
\left[1-(\alpha+\beta) x^{2}\right]-[1-\alpha] x=\frac{\alpha}{x}-\alpha+\beta
$$

which shows that the last inequality of (2.69) is equivalent to $\frac{\alpha}{x}<\alpha+\beta$. Since $x>0$, necessarily $\alpha+\beta>0$ and therefore, we have to find $x$ such that

$$
\left\{\begin{array}{l}
x>\frac{\alpha}{\alpha+\beta} \\
0<\alpha+\beta<1 \\
F(x)=0
\end{array}\right.
$$

It remains to find under what condition $F(x)$ admits a solution in the interval $] \frac{\alpha}{\alpha+\beta},+\infty[$.

From $\alpha+\beta<1$, we deduce that :

$$
\lim _{x \rightarrow+\infty} F(x)=+\infty
$$

Moreover : 


$$
F\left(\frac{\alpha}{\alpha+\beta}\right)=-\frac{\beta \alpha^{2}}{(\alpha+\beta)^{3}}
$$

Thus :

(i) If $\beta>0, P(x)$ admits at least one root in the interval $] \frac{\alpha}{\alpha+\beta},+\infty[(x=1$ is suitable since $\frac{\alpha}{\alpha+\beta}<1$ as soon as $\left.\beta>0\right)$.

(ii) If $\beta<0, \frac{\alpha}{\alpha+\beta}>1$. Let us remark that :

$$
F(x)=(x-1)\left\{[1-(\alpha+\beta)] x^{2}-\beta x+\alpha\right\}
$$

which clearly shows that $F(x)>0$ in the interval $] \frac{\alpha}{\alpha+\beta},+\infty[$.

Finally our method permits us to obtain a strong stability result if and only if :

$$
\left\{\begin{array}{l}
\alpha>0 \quad \beta>0 \\
0<\alpha+\beta<1
\end{array}\right.
$$

which are exactly the conditions we obtain by applying the Kreiss criterion. We have proved the following result:

\section{THEOREM 2.2}

Under the condition (2.49), the initial boundary value problem ((2.1), (2.48)) is strongly well-posed in the sense of Kreiss and the unique solution satisfies the identity (2.60) which yields the decay in time of the third order energy $E_{3}$ defined by (2.64). Moreover, this solution satisfies the a priori estimate:

$$
\sup _{|\alpha|=3}\left\|D^{\alpha} u\right\|_{L^{-}}\left(\mathbb{R}^{+} ; L^{2}(\Omega)\right) \leq C\left(\left\|u_{0}\right\|_{H^{3}(\Omega)}+\left\|u_{1}\right\|_{H^{2}(\Omega)}\right)
$$

where the positive constant $C$ only depends on $\gamma$ and $\beta$. 
It is interesting to notice that the equation $F(x)=0$ which appears naturally in this approach is nothing but the "characteristic equation" that one obtains when applying the normal mode analysis. Indeed, looking for generalized eigenvalues in the form :

$$
\left\{\begin{array}{l}
u(x, y, t)=\exp (-s t) \exp \xi y \text { exp ikx } \\
k \in \mathbb{R}, \mathscr{R e} \xi \geq 0
\end{array}\right.
$$

leads to the two equations :

$$
\begin{cases}\mathrm{k}^{2}=\xi^{2}-\mathrm{s}^{2} & \text { (interior wave equation) } \\ -\mathrm{s}^{3}+\mathrm{s}^{2} \xi-(\alpha+\beta) \mathrm{k}^{2} \mathrm{~s}+\alpha \mathrm{k}^{2} \xi=0 & \text { (boundary condition) }\end{cases}
$$

Eliminating $\mathrm{k}^{2}$ between these two equations is equivalent to eliminate the $\mathrm{x}$ derivatives in the boundary condition (2.47). We obtain the characteristic equation :

$$
[1-(\alpha+\beta)] s^{3}-(1-\alpha) s^{2} \xi+(\alpha+\beta) s \xi^{2}-\alpha \xi^{3}=0
$$

which is exactly $F(x)=0$ if we set $s=x \xi$.

\section{OBTENTION OF ENERGY TYPE A PRIORI ESTIMATES FOR THE WAVE EQUATION IN THE HALF-SPACE : THE GENERAL CASE}

We now consider general "absorbing" boundary conditions for the wave equation (2.1) obtained form the "approximation" of the function :

$$
f(s)=\sqrt{1-s^{2}}
$$

by the rational fraction :

$$
r(s)=\gamma-\sum_{k=1}^{N} \frac{\beta_{k} s^{2}}{1-\alpha_{k} s^{2}}
$$

where $\alpha_{k}, \beta_{k}$ and $\gamma$ are real numbers. The resulting boundary condition can be written in the Fourier domain $((x, t) \rightarrow(k, \omega))$ in the form : 


$$
\frac{\partial \widehat{u}}{\partial y}+i \omega \gamma \widehat{u}+i \omega \sum_{k=1}^{N} \frac{\beta_{k} k^{2}}{\omega^{2}-\alpha_{k} k^{2}} \widehat{u}=0
$$

Coming back to $(\mathrm{x}, \mathrm{t})$ variables, condition (3.2) can be reinterpreted as a system :

$$
\left\{\begin{array}{l}
\frac{\partial u}{\partial y}+\gamma \frac{\partial u}{\partial t}-\sum \beta_{k} \frac{\partial \varphi_{k}}{\partial t}=0 \\
\frac{\partial^{2} \varphi_{k}}{\partial t^{2}}-\alpha_{k} \frac{\partial^{2} \varphi_{k}}{\partial x^{2}}=\frac{\partial^{2} u}{\partial x^{2}}
\end{array}\right.
$$

or equivalently by :

$$
B_{2 N+1}\left(\frac{\partial}{\partial t}, \frac{\partial}{\partial x}, \frac{\partial}{\partial y}\right) u=0
$$

where the linear operator $\mathrm{B}_{2 \mathrm{~N}+1}$, differential operator of order $2 \mathrm{~N}+1$, is given by :

$$
\mid \begin{aligned}
B_{2 N+1}\left(\frac{\partial}{\partial t}, \frac{\partial}{\partial x}, \frac{\partial}{\partial y}\right) & =\left(\frac{\partial}{\partial y}+\gamma \frac{\partial}{\partial t}\right) \prod_{k=1}^{N}\left(\frac{\partial^{2}}{\partial t^{2}}-\alpha_{k} \frac{\partial^{2}}{\partial x^{2}}\right) \\
& -\frac{\partial}{\partial t} \sum_{k=1}^{N} \beta_{k} \frac{\partial^{2}}{\partial x^{2}}\left[\prod_{j \neq k}\left(\frac{\partial^{2}}{\partial t^{2}}-\alpha_{j} \frac{\partial^{2}}{\partial x^{2}}\right)\right]
\end{aligned}
$$

We can of course apply theorem A to obtain the necessary and sufficient conditions on the coefficients $\left(\gamma, \alpha_{j}, \beta_{k}\right)$ in order that the initial boundary value problem:

$$
\left\{\begin{array}{l}
\frac{\partial^{2} \mathrm{u}}{\partial t^{2}}-\Delta \mathrm{u}=0 \\
u(0)=\mathrm{u}_{0}, \frac{\partial \mathrm{u}}{\partial \mathrm{t}}(0)=\mathrm{u}_{1} \\
\mathrm{~B}_{2 \mathrm{~N}+1} \mathrm{u}=0
\end{array}\right.
$$

be well posed. These conditions are:

$$
\mid \begin{aligned}
& 0 \leq \alpha_{1}<\alpha_{2}<\cdots<\alpha_{N}<1 \\
& \beta_{k} \geq 0 \quad 1 \leq k \leq N \\
& \sum_{k=1}^{N} \frac{\beta_{k}}{1-\alpha_{k}}<\gamma
\end{aligned}
$$

Our goal in this section is to check that the same result can be obtained (and even improved in 
some sense) using energy techniques as we did for the second order and third order boundary conditions in sections 2.2 and 2.3. For technical reasons we shall decompose our analysis into two parts and consider separately :

\section{(i) The conditions of even order}

They correspond to $\alpha_{1}=0<\alpha_{2}$. We treat these conditions in section 3.2. As we shall see the second order boundary condition (2.19) treated in section (2.2.2) can be considered as a model for these conditions.

(ii) The conditions of odd order

They correspond to $\alpha_{1}>0$ and are treated in section 3.1. The third order boundary condition (2.47) is the model for these conditions.

The distinction between these two cases comes from the fact that in the case $\alpha_{1}=0$, the operator $B_{2 N+1}$, which is of order $2 N+1$, is a multiple of $\frac{\partial}{\partial t}$, in other words $B_{2 N+1}=\frac{\partial}{\partial t}\left(\widetilde{B}_{2 N}\right)$. Therefore, if we integrate (3.4) once in time (assuming that initial data vanish on the boundary), our boundary condition can be rewritten $\widetilde{\mathrm{B}}_{2 \mathrm{~N}} \mathrm{u}=0$ where now $\widetilde{\mathrm{B}}_{2 \mathrm{~N}}$ is an operator of order $2 \mathrm{~N}$.

For technical reasons, it seemed more natural to us to treat first the odd conditions. However, in [HJ2], we have adopted another approach to obtain the same conclusions as here. In particular, a link between the even and the odd conditions was presented in this paper.

\subsection{The case of odd order boundary conditions}

The first step of the analysis is to write a new boundary condition which is equivalent to (3.4) for any smooth solution of the wave equation. For this it suffices to replace formally $\frac{\partial^{2}}{\partial x^{2}}$ by $\frac{\partial^{2}}{\partial t^{2}}-\frac{\partial^{2}}{\partial y^{2}}$ in (3.4). So, one proves that any $C^{\infty}$-function u satisfying (3.4) on the boundary $\Gamma=\partial \Omega$, together with the wave equation (2.1) in the domain $\Omega$ also satisfies :

$$
B_{2 N+1}^{*} u=B_{2 N+1}^{*}\left(\frac{\partial}{\partial t}, \frac{\partial}{\partial y}\right) u=0
$$

where the linear differential operator $\mathrm{B}_{2 \mathrm{~N}+1}^{*}$ is given by : 
(3.9)

$$
\mid \begin{aligned}
& B_{2 N+1}^{*} u=B_{2 N+1}^{*}\left(\frac{\partial}{\partial t}, \frac{\partial}{\partial y}\right)=\frac{\partial Q}{\partial y}+\gamma \frac{\partial}{\partial t} Q-\frac{\partial}{\partial t} \sum_{k=1}^{N} \beta_{k}\left(\frac{\partial^{2}}{\partial t^{2}}-\frac{\partial^{2}}{\partial y^{2}}\right) \cdot Q_{k} \\
& Q=Q\left(\frac{\partial}{\partial t}, \frac{\partial}{\partial y}\right)=\prod_{k=1}^{N} P_{k} \\
& Q_{k}=Q_{k}\left(\frac{\partial}{\partial t}, \frac{\partial}{\partial y}\right)=\prod_{j+k} P_{j} \\
& P_{j}=P_{k}\left(\frac{\partial}{\partial t}, \frac{\partial}{\partial y}\right)=\alpha_{j} \frac{\partial^{2}}{\partial y^{2}}+\left(1-\alpha_{j}\right) \frac{\partial^{2}}{\partial t^{2}}
\end{aligned}
$$

In order to single out the principal part of $\mathrm{B}_{2 \mathrm{~N}+1}^{*}$ with respect to time, we remark that :

$$
\frac{\partial^{2}}{\partial t^{2}}-\frac{\partial^{2}}{\partial y^{2}}=\frac{1}{1-\alpha_{k}}\left\{P_{k}-\frac{\partial^{2}}{\partial y^{2}}\right\}
$$

so that we can also write :

$$
B_{2 N+1}^{*}=\frac{\partial Q}{\partial y}+\left[\gamma-\sum_{k=1}^{N} \frac{\beta_{k}}{1-\alpha_{k}}\right] \frac{\partial Q}{\partial t}+\sum_{k=1}^{N} \frac{\beta_{k}}{1-\alpha_{k}} \frac{\partial^{3} Q_{k}}{\partial y^{2} \partial t}
$$

Note that this expression is linked to the following expression for the rational fraction $r(s)$ :

$$
\mathrm{r}(\mathrm{s})=\left(\gamma-\sum_{\mathrm{k}=1}^{N} \frac{\beta_{\mathrm{k}}}{1-\alpha_{\mathrm{k}}}\right)-\sum_{\mathrm{k}=1}^{N} \frac{\beta_{\mathrm{k}}}{\alpha_{\mathrm{k}}} \frac{1}{1-\alpha_{\mathrm{k}} \mathrm{s}^{2}}
$$

Therefore, the boundary condition (3.8) can be rewritten as :

$$
-\frac{\partial}{\partial y}(Q u)=\left[\gamma-\sum_{k=1}^{N} \frac{\beta_{k}}{1-\alpha_{k}}\right] \frac{\partial Q u}{\partial t}+\sum_{k=1}^{N} \frac{\beta_{k}}{1-\alpha_{k}} \frac{\partial^{3}}{\partial y^{2} \partial t} Q_{k} u
$$

Now, we turn to the obtention of our main energy estimate. We first introduce :

$$
\mathrm{v}=\mathrm{Qu}
$$

Applying (2.4) to $\mathrm{v}$ leads to :

$$
\frac{d}{d t}\{E(v)\}=\int_{\Gamma} \frac{\partial Q u}{\partial y} \frac{\partial Q u}{\partial t} d \sigma
$$


Now, for $1 \leq k \leq N$, we introduce the functions :

$$
\left\{\begin{array}{l}
v_{k}=Q_{k} \frac{\partial^{2} u}{\partial y^{2}} \\
w_{k}=Q_{k} \frac{\partial^{2} u}{\partial y \partial t}
\end{array}\right.
$$

and apply (2.2) to $\mathrm{v}_{\mathrm{k}}$ and $\mathrm{w}_{\mathrm{k}}$. We obtain :

$$
\begin{aligned}
& \frac{d}{d t}\left\{E\left(v_{k}\right)\right\}=\int_{\Gamma} Q_{k} \frac{\partial^{3} u}{\partial y^{3}} \cdot Q_{k} \frac{\partial^{3} u}{\partial y^{2} \partial t} d \sigma \\
& \frac{d}{d t}\left\{E\left(w_{k}\right)\right\}=\int_{\Gamma} Q_{k} \frac{\partial^{3} u}{\partial y \partial t^{2}} \cdot Q_{k} \frac{\partial^{3} u}{\partial y^{2} \partial t} d \sigma
\end{aligned}
$$

We multiply (3.14) by $\alpha_{k},(3.15)$ by $\left(1-\alpha_{k}\right)$ and add the two resulting equalities. Using the fact that $\alpha_{k} \frac{\partial^{2}}{\partial y^{2}}+\left(1-\alpha_{k}\right) \frac{\partial^{2}}{\partial t^{2}}=P_{k}$ and that $P_{k} Q_{k}=Q$, we obtain :

$$
\frac{d}{d t}\left\{\alpha_{k} E\left(v_{k}\right)+\left(1-\alpha_{k}\right) E\left(w_{k}\right)\right\}=\int_{\Gamma} Q_{k} \frac{\partial^{3} u}{\partial y^{2} \partial t} \cdot \frac{\partial Q u}{\partial y} d \sigma
$$

Now we multiply (3.16) by $\frac{\beta_{\mathrm{k}}}{1-\alpha_{\mathrm{k}}}$, sum over $\mathrm{k}$ and add the result to (3.12) multiplied by $\left(\gamma-\sum_{\mathrm{k}=1}^{N} \frac{\beta_{\mathrm{k}}}{1-\alpha_{\mathrm{k}}}\right)$. This leads to :

$$
\begin{array}{|l}
\frac{d}{d t}\left\{\left(\gamma-\sum_{k=1}^{N} \frac{\beta_{k}}{1-\alpha_{k}}\right) E(v)+\sum_{k=1}^{N} \frac{\beta_{k} \alpha_{k}}{1-\alpha_{k}} E\left(v_{k}\right)+\sum_{k=1}^{N} \beta_{k} E\left(w_{k}\right)\right\} \\
=\int_{\Gamma} \frac{\partial Q u}{\partial y}\left(\left(\gamma-\sum_{k=1}^{N} \frac{\beta_{k}}{1-\alpha_{k}}\right) \frac{\partial Q u}{\partial t}+\sum_{k=1}^{N} \frac{\beta_{k}}{1-\alpha_{k}} \frac{\partial^{3}}{\partial y^{2} \partial t} Q_{k} u\right) d \sigma
\end{array}
$$

Using now (3.10), we finally get :

$$
\frac{d}{d t}\left\{E_{2 N+1}(u)\right\}=-\int_{\Gamma}\left|\frac{\partial}{\partial y}(Q u)\right|^{2} d \sigma<0
$$


where $E_{2 N+1}(u)$ denotes the $(2 N+1)^{\text {th }}$ order energy :

$$
E_{2 N+1}(u)=\left(\gamma-\sum_{k=1}^{N} \frac{\beta_{k}}{1-\alpha_{k}}\right) E(Q u)+\sum_{k=1}^{N} \frac{\beta_{k} \alpha_{k}}{1-\alpha_{k}} E\left(Q_{k} \frac{\partial^{2} u}{\partial y^{2}}\right)+\sum_{k=1}^{N} \beta_{k} E\left(Q_{k} \frac{\partial^{2} u}{\partial y \partial t}\right)
$$

As soon as the conditions (3.7) are satisfied all the coefficients appearing in the expression of $\mathrm{E}_{2 \mathrm{~N}+1}(\mathrm{u})$ are strictly positive and the fact that $\mathrm{E}_{2 \mathrm{~N}+1}(\mathrm{u})$ be a decreasing function of time leads to uniform bounds (by $\mathrm{C}\left(\left\|\mathrm{u}_{0}\right\|_{\mathrm{H}^{2 \mathrm{~N}+1}(\Omega)}+\left\|\mathrm{u}_{1}\right\|_{\mathrm{H}^{\mathrm{N}}(\Omega)}\right)$ in time of the $\mathrm{L}^{2}$-norms in space of the quantities :

$$
\mid \begin{array}{ll}
\frac{\partial}{\partial t}\left(Q_{u}\right), \frac{\partial}{\partial x}\left(Q_{u}\right), \frac{\partial}{\partial y}\left(Q_{u}\right) & \\
\frac{\partial}{\partial t}\left(Q_{k} \frac{\partial^{2} u}{\partial y^{2}}\right), \frac{\partial}{\partial x}\left(Q_{k} \frac{\partial^{2} u}{\partial y^{2}}\right), \frac{\partial}{\partial y}\left(Q_{k} \frac{\partial^{2} u}{\partial y^{2}}\right) & 1 \leq k \leq N \\
\frac{\partial}{\partial t}\left(Q_{k} \frac{\partial^{2} u}{\partial y \partial t}\right), \frac{\partial}{\partial x}\left(Q_{k} \frac{\partial^{2} u}{\partial y \partial t}\right), \frac{\partial}{\partial y}\left(Q_{k} \frac{\partial^{2} u}{\partial y \partial t}\right) & 1 \leq k \leq N
\end{array}
$$

Now, let $\mathscr{P}$ denote the set of polynomials of two variables $\mathrm{P}(\mathrm{s}, \xi)$ which are homogeneous and of degree $2 \mathrm{~N}$. $\mathscr{P}$ is a vector space of dimension $2 \mathrm{~N}+1$ and it is easy to check that it is generated by the $(2 \mathrm{~N}+1)$ polynomials :

$$
\begin{cases}Q_{(s, \xi)} & \\ s^{2} Q_{k}(s, \xi), & 1 \leq k \leq N \\ s \xi Q_{k}(s, \xi), & 1 \leq k \leq N\end{cases}
$$

Indeed assume that there exists $\left(\lambda, \lambda_{\mathrm{k}}, \mu_{\mathrm{k}}\right)$ such that :

$$
\lambda Q(s, \xi)+\sum_{k=1}^{N}\left\{\lambda_{k} s^{2} Q_{k}(s, \xi)+\mu_{k} s \xi Q_{k}(s, \xi)\right\}=0
$$

Choosing $s= \pm\left(\frac{\alpha_{k}}{1-\alpha_{k}}\right)^{1 / 2} \xi$ we simply obtain for each value of $k$ (we use the fact that all the $\alpha_{\mathrm{k}}$ are distinct so that $\mathrm{Q}_{\mathrm{k}}\left(\xi, \pm\left(\frac{\alpha_{\mathrm{k}}}{1-\alpha_{\mathrm{k}}}\right)^{1 / 2} \xi\right) \neq 0$ )

$$
\mu_{\mathrm{k}} \pm\left(\frac{\alpha_{\mathrm{k}}}{1-\alpha_{\mathrm{k}}}\right)^{1 / 2} \lambda_{\mathrm{k}}=0
$$

which implies that $\lambda_{\mathrm{k}}=\mu_{\mathrm{k}}=0$. Coming back to (3.22) we deduce that $\lambda=0$. This proves that the $(2 \mathrm{~N}+1)$ polynomials given by (3.21) are linearly independent and thus constitute a basis of $\mathscr{P}$. From (3.20) we deduce that the quantities : 


$$
\left.\mid \begin{array}{l}
\frac{\partial}{\partial t}\left(P\left(\frac{\partial}{\partial t}, \frac{\partial}{\partial y}\right) u\right) \\
\frac{\partial}{\partial x}\left(P\left(\frac{\partial}{\partial t}, \frac{\partial}{\partial y}\right) u\right) \\
\frac{\partial}{\partial y}\left(P\left(\frac{\partial}{\partial t}, \frac{\partial}{\partial y}\right) u\right)
\end{array}\right)
$$

are bounded in $\mathrm{L}^{\circ}\left(\mathbb{R}^{+} ; \mathrm{L}^{2}(\Omega)\right)$ for any $\mathrm{P}(.,$.$) in \mathscr{P}$ by $\mathrm{C}\left(\left\|\mathrm{u}_{0}\right\|_{\mathrm{H}^{2+1}(\Omega)}+\left\|\mathrm{u}_{1}\right\|_{\left.\mathrm{H}^{\mathrm{N}}(\Omega)\right) \text {. This implies }}\right.$ that

$$
\left\{\begin{array}{l}
\forall \alpha \in \mathbb{N}^{3} /|\alpha|=2 N+1 \\
D^{\alpha} u \text { is bounded in } L^{\infty}\left(\mathbb{R}^{+} ; L^{2}(\Omega)\right) \text { by } C\left(\left\|u_{0}\right\|_{H^{2 \mathbb{N}+1}(\Omega)}+\left\|u_{1}\right\|_{H^{\alpha}(\Omega)}\right)
\end{array}\right.
$$

Indeed any $D^{\alpha} \mathrm{u}$ containing at most first order derivative with respect to $\mathrm{x}$ can be written in the form (3.23). If derivatives of order higher than 2 with respect to $x$ appear, one can come back to the preceeding case by (repeated) use of the wave equation $\left(\frac{\partial^{2}}{\partial x^{2}}\right.$ can be replaced by $\left.\frac{\partial^{2}}{\partial t^{2}}-\frac{\partial^{2}}{\partial y^{2}}\right)$.

(3.24) is nothing but a strong stability result in the sense of Kreiss. Note that we can also derive boundary estimates from identity (3.17). For instance (3.17) directly provides that :

$$
\frac{\partial}{\partial y}(Q u)
$$

can be estimated in $\mathrm{L}^{2}\left(\mathbb{R}^{+} ; \mathrm{L}^{2}(\Gamma)\right)$.

Once again we see that for the $(2 \mathrm{~N}+1)^{\text {th }}$ order boundary condition (3.8), the energy one controls is a $(2 \mathrm{~N}+1)^{\text {th }}$ order energy, in the sense we define in section 1 , which means that it involves $(2 \mathrm{~N}+1)^{\text {th }}$ order derivatives of the solution. This is in fact coherent with the estimates one would obtain directly by the application of the Kreiss theory. Indeed if one wants to put the initial boundary problem for the wave equation coupled with condition (3.8) and the form of the first order systems studied by Kreiss, one has to introduce an unknown vector function $U$ of dimension $2 \mathrm{~N}+1$ whose coordinates are $2 \mathrm{~N}+1^{\text {th }}$ order derivatives of the function $u$. Therefore the $\mathrm{L}^{2}$-norm in space of $U$ corresponds to some energy of order $2 \mathrm{~N}+1$. Let us summarize the results of this section in the following theorem:

\section{THEOREM 3.1}

Under the condition (3.7), the initial boundary value problem $((2.1),(3.4))$ is strongly well-posed in 
the sense of Kreiss and the unique solution satisfies the identity (3.18) which yields the decay in time of the $(2 N+1)^{\text {th }}$ order energy $E_{2 N+1}$ defined by (3.19). Moreover, this solution satisfies the a priori estimate:

$$
\sup _{|\alpha|=2 N+1}\left\|D^{\alpha} u\right\|_{L-\left(R^{+} ; L^{2}(\Omega)\right)} \leq C\left(\left\|u_{0}\right\|_{H^{2 N+1}(\Omega)}+\left\|u_{1}\right\|_{H^{2 N}(\Omega)}\right)
$$

where the positive constant $C$ only depends on $\gamma, \alpha_{k}$ and $\beta_{k}, 1 \leq k \leq \mathrm{N}$.

\subsection{The case of the even order boundary conditions}

As we said before, the even order boundary conditions correspond to the particular case $\alpha_{1}=0$ so that in fact the a priori estimates we obtained in section 3.1, in particular the decay in time of the $(2 \mathrm{~N}+1)^{\text {th }}$ order $\mathrm{E}_{2 \mathrm{~N}+1}(\mathrm{u})$ given by (3.19) is still valid when we take $\alpha_{1}=0$. Nevertheless we prefere to treat separately the even order conditions becauce, since condition (3.4) for $\alpha_{1}=0$ corresponds in fact to a $2 \mathrm{~N}^{\text {th }}$ order condition, one can expect the decay in time of a $2 \mathrm{~N}^{\text {th }}$ order energy instead of a $(2 \mathrm{~N}+1)^{\text {th }}$ order one as $\mathrm{E}_{2 \mathrm{~N}+1}(\mathrm{u})$ is. Moreover this is more coherent with the definition of strong well-posedness by Kreiss.

Therefore the treatment of the conditions of even order will be slightly different from the one of the conditions of odd order. First we note, by similar arguments as in section 3.1 , that the condition (3.4) is equivalent to (we have integrated (3.4) once in time, which is possible since $\alpha_{1}=0$, and replaced $\frac{\partial^{2}}{\partial x^{2}}$ by $\left.\frac{\partial^{2}}{\partial t^{2}}-\frac{\partial^{2}}{\partial y^{2}}\right)$ :

$$
\mathrm{B}_{2 \mathrm{~N}}^{*} \mathrm{u}=\mathrm{B}_{2 \mathrm{~N}}^{*}\left(\frac{\partial}{\partial \mathrm{t}}, \frac{\partial}{\partial \mathrm{y}}\right) \mathrm{u}=0
$$

where the differential operator $\mathrm{B}_{2 \mathrm{~N}}^{*}$ is given by :

$$
\mathrm{B}_{2 \mathrm{~N}}^{*}=\left[\frac{\partial^{2}}{\partial y \partial t}+\gamma \frac{\partial^{2}}{\partial \mathrm{t}^{2}}\right] \mathrm{Q}_{1}-\sum_{\mathrm{k}=1}^{\mathrm{N}} \beta_{\mathrm{k}}\left[\frac{\partial^{2}}{\partial \mathrm{t}^{2}}-\frac{\partial^{2}}{\partial \mathrm{y}^{2}}\right] \mathrm{Q}_{\mathrm{k}}
$$

where the differential operators $\mathrm{Q}_{\mathrm{k}}$ have been defined in the previous section (simply note that in our case, $\mathrm{P}_{1}=\frac{\partial^{2}}{\partial \mathrm{t}^{2}}$ ). Using the identities : 


$$
\mid \begin{aligned}
& \frac{d}{d t}\left\{\left(\gamma-\sum_{k=1}^{N} \frac{\beta_{k}}{1-\alpha_{k}}\right) E\left(v_{1}\right)+\beta_{1} E\left(w_{1}\right)+\sum_{k=2}^{N}\left[\frac{\beta_{k} \alpha_{k}}{1-\alpha_{k}} E\left(v_{k}\right)+\beta_{k} E\left(w_{k}\right)\right]\right\} \\
& =\int_{\Gamma} \frac{\partial^{2}}{\partial y \partial t}\left(Q_{1} u\right) \cdot\left[\left(\gamma-\sum_{k=1}^{N} \frac{\beta_{k}}{1-\alpha_{k}}\right) \frac{\partial^{2}}{\partial t^{2}} Q_{1} u+\beta_{1} \frac{\partial^{2}}{\partial y^{2}} Q_{1} u+\sum_{k=2}^{N} \frac{\beta_{k}}{1-\alpha_{k}} \frac{\partial^{2}}{\partial y^{2}} Q_{k} u\right] d \sigma
\end{aligned}
$$

Thanks to (3.28), we finally obtain :

$$
\frac{d}{d t}\left\{E_{2 N}(u)\right\}=-\int_{\Gamma}\left|\frac{\partial^{2}}{\partial y \partial t}\left(Q_{1} u\right)\right|^{2} d \sigma
$$

where $E_{2 N}(u)$ denotes the $2 N^{\text {th }}$ order energy :

$$
\mid \begin{aligned}
E_{2 N}(u) & =\left(\gamma-\sum_{k=1}^{N} \frac{\beta_{k}}{1-\alpha_{k}}\right) E\left(\frac{\partial}{\partial t} Q_{1} u\right)+\beta_{1} E\left(\frac{\partial}{\partial y} Q_{1} u\right) \\
& +\sum_{k=2}^{N}\left\{\frac{\beta_{k} \alpha_{k}}{1-\alpha_{k}} E\left(\frac{\partial^{3}}{\partial y^{2} \partial t} q_{k} u\right)+\beta_{k} E\left(\frac{\partial^{3}}{\partial y \partial t^{2}} q_{k} u\right)\right\}
\end{aligned}
$$

It is then easy to check that, as in section 3.1 , identity (3.40) is equivalent, as soon as the stability conditions (3.7) are fullfilled, to a strong stability result. In fact it suffices to verify that the $2 N$ operators $\frac{\partial}{\partial t} Q_{1}, \frac{\partial}{\partial y} Q_{1}$ and $\left\{\frac{\partial^{3}}{\partial \dot{y}^{2} \partial t} q_{k}, \frac{\partial^{3}}{\partial y \partial t^{2}} q_{k}, 2 \leq k \leq N\right\}$ generate all the homogeneous differential operators of order $2 \mathrm{~N}-1$ with respect to $y$ and $t$. The details are left to the reader. One finally obtains:

\section{THEOREM 3.2}

Under the condition (3.7), the initial boundary value problem $((2.1),(3.25))$ is strongly well-posed in the sense of Kreiss and the unique solution satisfies the identity (3.39) which yields the decay in time of the $2 N^{\text {th }}$ order energy $E_{2 N}$ defined by (3.40). Moreover, this solution satisfies the a priori estimate:

$$
\sup _{|\alpha|=2 N}\left\|D^{\alpha}\right\|_{L} \|_{L^{2}\left(R^{+} ; L^{2}(\Omega)\right)} \leq C\left(\left\|u_{0}\right\|_{H^{2}(\Omega)}+\left\|u_{1}\right\|_{\left.H^{2 N-1}(\Omega)\right)}\right.
$$

where the positive constant $C$ only depends on $\gamma, \alpha_{k}$ and $\beta_{k}, 1 \leq k \leq \mathrm{N}$. 


\section{CONCLUSION}

In this paper, we have revisited the theory of the strong well-posedness of initial boundary value problems for the wave equations via the approach of energy estimates, which is, up to our knowledge, new. This method allowed us to find again the conditions obtained by Trefethen-Halpern in the framework of the modal analysis and to improve the a priori estimates directly deduced from the theory of Kreiss.

Moreover, the second interest of the approach lies in the fact that we shall be able to extend some strong stability results to the case of variable coefficients even if these coefficients are not smooth. This will be the purpose of a forthcoming paper. 


\section{REFERENCES :}

[EM] ENGQUIST B., MAJDA A., Radiation boundary conditions for acoutic and elastic wave calculations, Comm. Pure Appl. Math., v.32, 1979, pp. 313-357.

[F] FRIEDRICHS K.O., Symmetric hyperbolic linear partial differential equations, Comm. Pure Appl. Math., v.7, 1954, pp. 345, 394.

[GT] GOLDBERG M., TADMOR E., Convenient stability criteria for difference approximations of hyperbolic initial boundary value problems, Math. of Comp., v.44, 1985, pp. 361-377.

[GKS] GUSTAFSSON B., KREISS H. O. AND SUNDSTRÖM A., Stability theory of difference approximations for mixed initial boundary value problems $\Pi$, Math. of Comp., v.25, 1972, pp. 649-686.

[He] HERSCH R., Mixed problems in several variables, J. Math. Mech., v.12, 1963, pp 317-334.

[HJ] HADUONG T., JOLY P., A generalized image principle for the wave equation with absorbing boundary conditions and applications to fourth order schemes, INRIA report, to appear 1990 (submitted to Math. of Comp.)

[HJ2] HADUONG T., JOLY P., Energy identities for the wave equation with high order boundary conditions, submitted to the First International Conference on Wave Propagation, Strasbourg 1991.

[Hi] HIGDON R.L., Initial boudary value problems for linear hyperbolic systems, SIAM Rev., v.28, 1986, pp. 177-217.

[Hi2] HIGDON R.L., Absorbing boundary conditions for difference approximations to the multidimensional wave equation, Math. of Comp., v.47, 1986, pp. 437-460.

[K] KREISS H.O.., Initial boundary value problems for hyperbolic systems. Comm. Pure Appl. Math., v.23, 1970, pp. 277-298.

[LM] LIONS J.L., MAGENES E., Problèmes aux limites non homogènes et applications, Dunod, Paris 1968. 
[MO] MAJDA A., OSHER S., Initial boundary value problems for hyperbolic equations with uniformly characteristic boundary, Comm. Pure Appl. Math., v.28, 1975, pp. 607-675.

[M] MICHELSON D., Stability theory of difference approximations for multidimensional initial boundary value problems, Math. of Comp., v.40, 1983, pp. 1-45.

[T] TREFETHEN L.N., Instability of difference models for hyperbolic initial boundary value problems, Comm. Pure Appl. Math., v.37, 1984, pp. 329-367.

[TH] TREFETHEN L.N., HALPERN L., Well-posedness of one way wave equations and absorbing boundary conditions. Math. of Comp., v.47, 1986, pp. 421-435.

[W] WILCOX C., Asymptotic wave functions and energy distributions in strongly propagative anisotropic media, J. Math. Pures et Appl., v.57, 1978, pp. 275-321. 

ISSN 0249 - 6399 